Homology, Homotopy and Applications, vol.18(1), 2016, pp.71-98

\title{
BIMODULES AND NATURAL TRANSFORMATIONS FOR ENRICHED $\infty$-CATEGORIES
}

\author{
RUNE HAUGSENG
}

(communicated by Emily Riehl)

\begin{abstract}
We introduce a notion of bimodule in the setting of enriched $\infty$-categories, and use this to construct a double $\infty$-category of enriched $\infty$-categories where the two kinds of 1-morphisms are functors and bimodules. We then consider a natural definition of natural transformations in this context, and show that in the underlying $(\infty, 2)$-category of enriched $\infty$-categories with functors as 1-morphisms the 2-morphisms are given by natural transformations.
\end{abstract}

\section{Introduction}

This paper is a sequel to [GH15] and part of [Hau14b]: In [GH15], David Gepner and I set up a theory of enriched $\infty$-categories, using a non-symmetric variant of Lurie's theory of $\infty$-operads, and in [Hau14b, §4] I constructed a double $\infty$-category $\mathfrak{A} \mathfrak{L} \mathfrak{G}(\mathcal{V})$ of associative algebra objects in a monoidal $\infty$-category $\mathcal{V}$, with the two kinds of 1-morphism given by algebra homomorphisms and bimodules. The goal of this paper is to construct a "many-object" analogue of this double $\infty$-category: In [GH15] we defined enriched $\infty$-categories as algebras for "many-object associative operads", and there is an analogous extension of the definition of bimodules in [Hau14b] using "many-object bimodule operads". Using this definition we extend the constructions of $[\mathrm{Hau} \mathbf{1 4 b}]$ to get our main result:

Theorem 1.1. Let $\mathcal{V}$ be a monoidal $\infty$-category compatible with small colimits. Then there exists a double $\infty$-category $\mathfrak{A} \mathfrak{L G}_{\text {cat }}(\mathcal{V})$ of $\mathcal{V}$-enriched $\infty$-categories, with the two kinds of 1-morphism given by bimodules and functors. Moreover, if $\mathcal{V}$ is an $\mathbb{E}_{n+1^{-}}$ monoidal $\infty$-category, then $\mathfrak{A} \mathfrak{L G}_{\text {cat }}(\mathcal{V})$ inherits a natural $\mathbb{E}_{n}$-monoidal structure.

We'll construct this double $\infty$-category in $\S 6$ and discuss its functoriality and monoidal structures in $\S 9$.

We can also restrict the objects of this double $\infty$-category to those $\mathcal{V}$ - $\infty$-categories that are complete, i.e. local with respect to the fully faithful and essentially surjective

Received June 28, 2015, revised August 13, 2015; published on March 7, 2016.

2010 Mathematics Subject Classification: 18D05, 18D20, 55U40.

Key words and phrases: enriched $\infty$-category, bimodule.

Article available at http://dx.doi.org/10.4310/HHA.2016.v18.n1.a5

Copyright (C) 2016, Rune Haugseng. Permission to copy for private use granted. 
functors, to obtain the double $\infty$-category $\mathfrak{C A T}(\mathcal{V})$, which we regard as the "correct" double $\infty$-category of $\mathcal{V}$ - $\infty$-categories. ${ }^{1}$

The double $\infty$-category $\mathfrak{A L G}_{\text {cat }}(\mathcal{V})$ has two underlying $(\infty, 2)$-categories, with the 1-morphisms given either by bimodules or by functors. In the latter case, we would expect the 2-morphisms to be natural transformations. The second main result of this paper is that this is indeed the case: We will use the obvious notion of a natural transformation of functors between $\mathcal{V}$ - $\infty$-categories $\mathcal{C}$ and $\mathcal{D}$, namely a functor

$$
\mathcal{C} \otimes[1] \rightarrow \mathcal{D}
$$

to define a Segal space $\operatorname{Fun}_{\mathcal{V}}(\mathcal{C}, \mathcal{D})$ of $\mathcal{V}$-functors, and show:

Theorem 1.2. Let $\mathrm{ALG}_{\mathrm{cat}}(\mathcal{V})$ be the $(\infty, 2)$-category (in the sense of a 2-fold Segal space) underlying $\mathfrak{A} \mathfrak{L G}_{\text {cat }}(\mathcal{V})$ with functors as 1-morphisms. There is a natural equivalence between $\operatorname{Fun}_{\mathcal{V}}(\mathcal{C}, \mathcal{D})$ and the Segal space $\operatorname{ALG}_{\text {cat }}(\mathcal{V})(\mathcal{C}, \mathcal{D})$ of maps from $\mathcal{C}$ to $\mathcal{D}$ in $\mathfrak{A} \mathfrak{L} \mathfrak{G}_{\text {cat }}(\mathcal{V})$.

We'll prove this in $\S 8$. If $\mathcal{D}$ is complete we will also observe that the Segal space $\operatorname{Fun}_{\mathcal{V}}(\mathcal{C}, \mathcal{D})$ is complete for any $\mathcal{C}$, so as a consequence we obtain that the 2-fold Segal space $\mathrm{CAT}_{\infty}^{\mathcal{V}}$ underlying $\mathfrak{C A T}(\mathcal{V})$ with functors as 1-morphisms is complete.

In ordinary enriched category theory the notion of bimodule is classical, and according to the nlab was invented independently by a number of people back in the 1960s, though with much of their theory introduced by Bénabou. The specific definition of a bimodule between enriched $\infty$-categories we consider here was, however, inspired by the "external" notion of bimodule given by Bacard in [Bac10] in the context of a model-categorical approach to weakly enriched categories.

To motivate this paper, let's now briefly consider some future directions in which I hope to extend the results proved here:

- In [Hau14b, §6] I constructed for any $\mathbb{E}_{n}$-monoidal $\infty$-category an $(\infty, n+1)$ category of $\mathbb{E}_{n}$-algebras in it. Similarly, I hope to construct $(\infty, n+1)$-categories of enriched $(\infty, n)$-categories also for $n>1$ - these are expected to be the targets for a number of interesting extended topological quantum field theories.

- In $[$ Lur14, §4.6.3] Lurie proves that all associative algebras are dualizable in the $\infty$-category of algebras and bimodules. This should extend to a proof that all enriched $\infty$-categories are dualizable, which will lead to a definition of topological Hochschild homology for enriched $\infty$-categories. Similarly, the proof in [Lur14, $\S 4.6 .4]$ that the 2-dualizable algebras are precisely the smooth and proper ones should extend to a characterization of the 2 -dualizable enriched $\infty$-categories.

- For ordinary enriched categories, a bimodule between $\mathbf{V}$-categories $\mathbf{C}$ and $\mathbf{D}$ is often defined as a functor from $\mathbf{C} \otimes \mathbf{D}^{\mathrm{op}}$ to the self-enrichment of $\mathbf{V}$. The same should be true for the bimodules we consider here: the $\infty$-category of $\mathcal{C}$ $\mathcal{D}$-bimodules in $\mathcal{V}$ should be a representable functor of $\mathcal{C}$, with the representing object being $\mathcal{V}$-valued enriched presheaves on $\mathcal{D}$. This can be thought of as a form of the Yoneda Lemma for enriched $\infty$-categories. (In particular, the more

\footnotetext{
${ }^{1}$ However, we do not show here that this double subcategory is functorial or inherits the monoidal structures on $\mathfrak{A} \mathfrak{L} \mathfrak{G}_{\text {cat }}(\mathcal{V})$ - this is a consequence of the Yoneda Lemma, which we hope to prove in a sequel to this paper.
} 
obvious formulation that there is a fully faithful Yoneda embedding into enriched presheaves would be an easy consequence of this.)

- Classically, the double category of V-enriched categories, functors, and bimodules is an example of a proarrow equipment. This is an abstract context in which one can define weighted (co)limits and Kan extensions. An analogous theory can be developed in the $\infty$-categorical context, with the double $\infty$-category we construct here as a key example. Combined with the Yoneda Lemma, which gives a checkable criterion for a bimodule to be represented by a functor, this should give very useful tools for making interesting constructions and in general "doing category theory" with enriched $\infty$-categories (with a particularly interesting case here being $(\infty, n)$-categories).

\subsection{Overview}

In $\S 2$ we review some key notions and results from the theory of non-symmetric $\infty$-operads, and in $\S 3$ we briefly recall the main definitions and results on enriched $\infty$ categories from [GH15] that we'll make use of. Then in $\S 4$ we introduce our definition of bimodules between enriched $\infty$-categories, and motivate it by relating it to the classical notion of a bimodule for enriched categories. Next we discuss, in $\S 5$, how to compose these bimodules, and observe that this is analogous to the composition of bimodules for ordinary enriched categories. After these introductory sections we then get to work in $\S 6$, where we construct the double $\infty$-category of enriched $\infty$-categories. In $\S 7$ we consider the obvious definition of natural transformations in this context and show these are the 1-morphisms in an $\infty$-category of enriched functors, and then we compare this to the mapping $\infty$-category of functors coming from our double $\infty$ category in $\S 8$. Finally we discuss the functoriality of the double $\infty$-categories and their natural monoidal structures in $\S 9$.

\subsection{Notation}

We recycle the notation of [GH15] and [Hau14b]. In particular, for $[n]$ an object of $\triangle$ we'll abbreviate $\left(\Delta_{/[n]}\right)^{\text {op }}$ to $\triangle_{/[n]}^{\mathrm{op}}$ to avoid clutter as this object will appear frequently, often in subscripts. If $\phi:[m] \rightarrow[n]$ is an object of $\Delta_{/[n]}$ we'll also denote this object by the list $(\phi(0), \ldots, \phi(m))$ where $0 \leqslant \phi(i) \leqslant \phi(i+1) \leqslant m$.

\section{Acknowledgments}

This is the final paper based on part of my Ph.D. thesis - though much improved by being left to stew for a while - so it is a pleasure to get to thank Haynes Miller once more for being a great advisor, as well as the Norway-America Association and the American-Scandinavian Foundation for partially funding my studies at MIT. This also seems an appropriate occasion to thank David Gepner for steering me away from a truly atrocious approach to defining $\infty$-categories of functors between enriched $\infty$-categories back in 2012 .

\section{Non-symmetric $\infty$-operads}

Here we briefly recall some of the basic definitions from the theory of (non-sym- 
metric) $\infty$-operads and summarize some key results that we will use in this paper. For motivation for these definitions we refer the reader to the discussion in [GH15, §2], and for proofs we refer to [GH15, $\S 3$ and $\S \mathrm{A}]$, and of course [Lur14].

Definition 2.1. Let $\triangle$ be the usual simplicial indexing category. A morphism $f:[n] \rightarrow[m]$ in $\Delta$ is inert if it is the inclusion of a sub-interval of $[m]$, i.e. $f(i)=$ $f(0)+i$ for all $i$, and active if it preserves the extremal elements, i.e. $f(0)=0$ and $f(n)=m$. We say a morphism in $\Delta^{\mathrm{op}}$ is active or inert if it is so when considered as a morphism in $\triangle$, and write $\triangle_{\text {act }}^{o p}$ and $\triangle_{\text {int }}^{o p}$ for the subcategories of $\Delta^{\text {op }}$ with active and inert morphisms, respectively. We write $\rho_{i}:[n] \rightarrow[1]$ for the inert map in $\Delta^{\mathrm{op}}$ corresponding to the inclusion $\{i-1, i\} \hookrightarrow[n]$.

Definition 2.2. A generalized non-symmetric $\infty$-operad is an inner fibration $\pi: \mathcal{M} \rightarrow \triangle^{\mathrm{op}}$ such that:

(i) For each inert map $\phi:[n] \rightarrow[m]$ in $\triangle^{\text {op }}$ and every $X \in \mathcal{M}$ such that $\pi(X)=[n]$, there exists a $\pi$-coCartesian edge $X \rightarrow \phi_{!} X$ over $\phi$.

(ii) For every $[n]$ in $\triangle^{\mathrm{op}}$, the map

$$
\mathcal{M}_{[n]} \rightarrow \mathcal{M}_{[1]} \times \mathcal{M}_{[0]} \cdots \times \times_{\mathcal{M}_{[0]}} \mathcal{M}_{[1]}
$$

induced by the inert maps $[n] \rightarrow[1],[0]$ is an equivalence.

(iii) Given $C \in \mathcal{M}_{[n]}$ and a coCartesian map $C \rightarrow C_{\alpha}$ over each inert map $\alpha$ from $[n]$ to $[1]$ and [0], the object $C$ is a $\pi$-limit of the $C_{\alpha}$ 's.

A non-symmetric $\infty$-operad is a generalized non-symmetric $\infty$-operad $\mathcal{M}$ such that $\mathcal{M}_{0} \simeq *$.

Definition 2.3. A double $\infty$-category is a generalized non-symmetric $\infty$-operad $\mathcal{M} \rightarrow$ $\triangle^{\text {op }}$ that is also a coCartesian fibration, and a monoidal $\infty$-category is a non-symmetric $\infty$-operad that is also a coCartesian fibration.

Equivalently, a double $\infty$-category can be defined as a coCartesian fibration such that the associated functor $F: \triangle^{\mathrm{op}} \rightarrow \mathrm{Cat}_{\infty}$ satisfies the Segal condition: for every $[n] \in \triangle^{\mathrm{op}}$, the functor

$$
F([n]) \rightarrow F([1]) \times_{F([0])} \cdots \times_{F([0])} F([1])
$$

induced by the maps $\rho_{i}:[n] \rightarrow[1]$ and all the maps $[n] \rightarrow[0]$, is an equivalence of $\infty$-categories.

Definition 2.4. A morphism of (generalized) non-symmetric $\infty$-operads is a commutative diagram

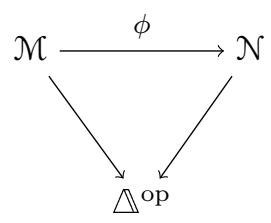

such that $\phi$ carries inert morphisms in $\mathcal{M}$ to inert morphisms in $\mathcal{N}$. We will also refer to a morphism of (generalized) non-symmetric $\infty$-operads $\mathcal{N} \rightarrow \mathcal{N}$ as an $\mathcal{M}$-algebra in $\mathcal{N}$; we write $\operatorname{Alg}_{\mathcal{M}}(\mathcal{N})$ for the full subcategory of the $\infty$-category $\operatorname{Fun}_{\triangle^{\text {op }}}(\mathcal{M}, \mathcal{N})$ 
of functors over $\triangle^{\text {op }}$ spanned by the morphisms of (generalized) non-symmetric $\infty$ operads.

Using the theory of categorical patterns developed in [Lur14, §B], we can define $\infty$-categories $\mathrm{Opd}_{\infty}^{\text {ns }}$ and $\mathrm{Opd}_{\infty}^{\text {ns, gen }}$ of non-symmetric $\infty$-operads and generalized nonsymmetric $\infty$-operads. The $\infty$-categories of algebras are functorial in these $\infty$-categories, and indeed determine a lax monoidal functor

$$
\left(\mathrm{Opd}_{\infty}^{\mathrm{ns}, \mathrm{gen}}\right)^{\mathrm{op}} \times \mathrm{Opd}_{\infty}^{\mathrm{ns}, \text { gen }} \rightarrow \mathrm{Cat}_{\infty}
$$

For a generalized non-symmetric $\infty$-operad $\mathcal{M}$ we define the algebra fibration

$$
\operatorname{Alg}(\mathcal{M}) \rightarrow \mathrm{Opd}_{\infty}^{\mathrm{ns}, \mathrm{gen}}
$$

to be a Cartesian fibration associated to the functor

$$
\operatorname{Alg}_{(-)}(\mathcal{M}):\left(\operatorname{Opd}_{\infty}^{\mathrm{ns}, \text { gen }}\right)^{\text {op }} \rightarrow \mathrm{Cat}_{\infty}
$$

We say that a monoidal $\infty$-category $\mathcal{V}^{\otimes}$ is compatible with small colimits if the underlying $\infty$-category $\mathcal{V}$ has small colimits and the tensor product preserves colimits in each variable. If $\mathcal{V}$ is compatible with small colimits and $f: \mathcal{O} \rightarrow \mathcal{P}$ is a morphism of small $\infty$-operads, then the functor $f^{*}: \operatorname{Alg}_{\mathcal{P}}(\mathcal{V}) \rightarrow \operatorname{Alg}_{\mathcal{O}}(\mathcal{V})$ has a left adjoint $f_{!}$, given by taking operadic left Kan extensions along $f$. If $A$ is an $\mathcal{O}$-algebra in $\mathcal{V}$, the value of the $\mathcal{P}$-algebra $f_{!} A$ at $p \in \mathcal{P}_{[1]}$ is given by a certain colimit, ${ }^{2}$ which we can somewhat informally express as

$$
\underset{\left(o_{1}, \ldots, o_{n}\right) \in \mathcal{O}_{/ p}^{\text {act }}}{\operatorname{colim}} A\left(o_{1}\right) \otimes \cdots \otimes A\left(o_{n}\right) .
$$

Here $\mathcal{O}_{/ p}^{\text {act }}$ is the $\infty$-category $\mathcal{O}^{\text {act }} \times$ pact $\mathcal{P}_{/ p}^{\text {act }}$ of objects of $\mathcal{O}$ whose image in $\mathcal{P}$ has an active map to $p$, and active maps between them.

In good cases we can also explicitly describe this left adjoint in the same way when $f$ is a morphism of generalized non-symmetric $\infty$-operads (by [Hau14b, Corollary A.60]), namely if $f$ is extendable in the sense of [Hau14b, Definition A.49], which we recall here for the reader's convenience:

Definition 2.5. A morphism $f: \mathcal{O} \rightarrow \mathcal{P}$ of generalized non-symmetric $\infty$-operads is extendable if for every $P \in \mathcal{P}$ lying over $[n] \in \mathbb{\triangle}^{\mathrm{op}}$, the morphism

$$
\mathcal{O}_{/ P}^{\text {act }} \rightarrow \prod_{i=1}^{n} \mathcal{O}_{/ P_{i}}^{\text {act }}
$$

induced by the coCartesian morphisms in $\mathcal{O}$ over the maps $\rho_{i}$, is cofinal.

\section{Enriched $\infty$-categories}

In this section we recall the definition of enriched $\infty$-categories as "many-object associative algebras" we introduced in [GH15], and some key definitions and results

\footnotetext{
${ }^{2}$ In fact, by [Lur14, Corollary 3.1.3.5] this colimit description essentially characterizes $f_{!} A$, in the sense that a morphism $\phi: A \rightarrow f^{*} B$ is adjunct to an equivalence $f ! A \stackrel{\sim}{\rightarrow} B$ if and only if $\phi$ induces an equivalence between the appropriate colimit and $B(p)$ for all $p \in \mathcal{P}_{[1]}$.
} 
from that paper that we will make use of here. For further motivation for this definition we refer to $[\mathbf{G H 1 5}, \S 2]$, and for complete details of the constructions we refer to $[\mathrm{GH} 15, \S 4-5]$.

Definition 3.1. Given a space $X$, let $i_{X}: \triangle^{\mathrm{op}} \rightarrow \mathcal{S}$ be the right Kan extension of the functor $\{[0]\} \rightarrow \mathcal{S}$ that sends $[0]$ to $X$. We write $\triangle_{X}^{\mathrm{op}} \rightarrow \Delta^{\mathrm{op}}$ for the left fibration associated to the functor $i_{X}$.

Remark 3.2. The functor $i_{X}$ sends $[n]$ to $X^{\times(n+1)}$ and takes face maps to projections and degeneracies to diagonal maps. We can thus identify the objects of $\Delta_{X}^{\mathrm{op}}$ lying over $[n]$ with lists $\left(x_{0}, \ldots, x_{n}\right)$ of points $x_{i} \in X$.

Lemma 3.3 ([GH15, Lemma 4.1.3]). For any space $X$, the projection $\triangle_{X}^{\mathrm{op}} \rightarrow \triangle^{\mathrm{op}}$ is a double $\infty$-category.

Definition 3.4. If $\mathcal{V}$ is a monoidal $\infty$-category, a $\mathcal{V}$-enriched $\infty$-category (or $\mathcal{V}$ - $\infty$ category) with space of objects $X$ is a $\triangle_{X}^{\text {op }}$-algebra in $\mathcal{V}$.

Remark 3.5. Observe that an algebra $\mathcal{C}: \triangle_{X}^{\text {op }} \rightarrow \mathcal{V}^{\otimes}$ assigns to every $(x, y) \in X^{\times 2}$ an object $\mathrm{C}(x, y) \in \mathcal{V}$. Speaking slightly informally, for every $(x, y, z) \in X^{\times 3}$ the face map $(x, y, z) \rightarrow(x, z)$ in $\triangle_{X}^{\text {op }}$ gives a composition map $\mathcal{C}(x, y) \otimes \mathcal{C}(y, z) \rightarrow \mathcal{C}(x, z)$, and the degeneracy $(x) \rightarrow(x, x)$ gives a unit $I \rightarrow \mathfrak{C}(x, x)$ where $I$ is the unit of the monoidal $\infty$-category $\mathcal{V}$. The remaining data in $\mathcal{C}$ shows that this composition is coherently homotopy-associative and compatible with the unit - this is precisely the data we would expect an enriched $\infty$-category to be given by.

Definition 3.6. The $\infty$-categories $\triangle_{X}^{\text {op }}$ are clearly functorial in $X$, and so determine a functor $\mathcal{S} \rightarrow \operatorname{Opd}_{\infty}^{\text {ns,gen }}$. If $\mathcal{V}$ is a monoidal $\infty$-category, we let $\operatorname{Alg}_{\text {cat }}(\mathcal{V}) \rightarrow \mathcal{S}$ be a Cartesian fibration associated to the functor $\mathcal{S}^{\mathrm{op}} \rightarrow \operatorname{Cat}_{\infty}$ that sends $X$ to $\operatorname{Alg}_{\triangle_{X}^{\mathrm{op}}}(\mathcal{V})$.

If we take $\mathcal{V}$ to be the $\infty$-category $\mathcal{S}$ of spaces, then $\operatorname{Alg}_{\text {cat }}(\mathcal{S})$ is equivalent to the $\infty$-category $\mathrm{Seg}_{\infty}$ of Segal spaces.

The $\infty$-category $\operatorname{Alg}_{\text {cat }}(\mathcal{V})$ is functorial in $\mathcal{V}$, and it is a lax monoidal functor with respect to the Cartesian product of monoidal $\infty$-categories and of $\infty$-categories. Moreover, if $\mathcal{V}$ is compatible with small colimits then $\operatorname{Alg}_{\text {cat }}(\mathcal{V})$ has small colimits, and it is tensored over $\operatorname{Alg}_{\text {cat }}(\mathcal{S})$ in such a way that the tensoring preserves colimits in each variable. Any Segal space thus gives rise to a $\mathcal{V}$ - $\infty$-category by tensoring with the unit of $\mathcal{V}$, regarded as a 1 -object $\mathcal{V}$ - $\infty$-category.

In particular, if we write $E^{n}$ for the contractible category with objects $\{0, \ldots, n\}$ and a unique morphism $i \rightarrow j$ for all $i$ and $j$, this determines a $\mathcal{V}$ - $\infty$-category we also denote $E^{n}{ }^{3}$ We say that a $\mathcal{V}$ - $\infty$-category $\mathcal{C}$ is complete if it is local with respect to the map $E^{1} \rightarrow E^{0}$, i.e. if the map of $\operatorname{spaces} \operatorname{Map}\left(E^{0}, \mathcal{C}\right) \rightarrow \operatorname{Map}\left(E^{1}, \mathcal{C}\right)$ is an equivalence. Under the equivalence between $\operatorname{Alg}_{\text {cat }}(\mathcal{S})$ and Segal spaces, the complete $\mathcal{S}$ - $\infty$-categories precisely correspond to the complete Segal spaces in the sense of Rezk.

We write $\mathrm{Cat}_{\infty}^{\mathcal{V}}$ for the full subcategory of $\operatorname{Alg}_{\text {cat }}(\mathcal{V})$ spanned by the complete $\mathcal{V}$ $\infty$-categories. Our main result in $\left[\right.$ GH15] was that the inclusion $\operatorname{Cat}_{\infty}^{\mathcal{V}} \hookrightarrow \operatorname{Alg}_{\text {cat }}(\mathcal{V})$ has a left adjoint, which exhibits $\mathrm{Cat}_{\infty}^{\mathcal{V}}$ as the localization of $\operatorname{Alg}_{\text {cat }}(\mathcal{V})$ with respect

${ }^{3}$ In fact, we can define $E^{n}$ as a $\mathcal{V}$ - $\infty$-category for an arbitrary $\mathcal{V}$, but we will not need this generality here. 
to the class of fully faithful and essentially surjective morphisms. ${ }^{4}$ This means that Cat $_{\infty}^{\mathcal{V}}$ is the "correct" homotopy theory of $\mathcal{V}$-enriched $\infty$-categories.

\section{Bimodules}

If $\mathbf{V}$ is a closed symmetric monoidal category, so that there is a tensor product of $\mathbf{V}$-categories and $\mathbf{V}$ has a natural self-enrichment $\overline{\mathbf{V}}$, the classical definition of a bimodule between $\mathbf{V}$-categories $\mathbf{C}$ and $\mathbf{D}$ is a $\mathbf{V}$-functor

$$
M: \mathbf{C}^{\mathrm{op}} \otimes \mathbf{D} \rightarrow \overline{\mathbf{V}} .
$$

We can reformulate this definition to see it as a many-object version of the usual notion of a bimodule for associative algebras: unravelling the definition, a C-Dbimodule consists of:

- for all $c \in \mathbf{C}$ and $d \in \mathbf{D}$, an object $M(c, d) \in \mathbf{V}$,

- for all $c^{\prime}, c \in \mathbf{C}$ and $d \in \mathbf{D}$, an action map $\mathbf{C}\left(c^{\prime}, c\right) \otimes M(c, d) \rightarrow M\left(c^{\prime}, d\right)$, compatible with composition and units in $\mathbf{C}$,

- for all $c \in \mathbf{C}$ and $d, d^{\prime} \in \mathbf{D}$, an action map $M(c, d) \otimes \mathbf{D}\left(d, d^{\prime}\right) \rightarrow M\left(c, d^{\prime}\right)$, compatible with composition and units in $\mathbf{D}$,

such that for $c, c^{\prime} \in \mathbf{C}$ and $d, d^{\prime} \in \mathbf{D}$, the diagram

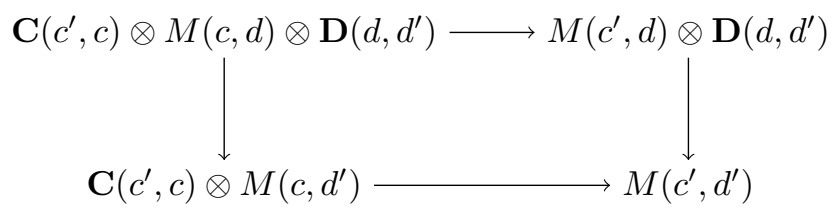

commutes. Notice that this definition does not require $\mathbf{V}$ to be closed or symmetric monoidal.

Since we defined enriched $\infty$-categories as algebras for "many-object associative $\infty$-operads", this suggests that we can define bimodules for enriched $\infty$-categories as algebras for "many-object bimodule $\infty$-operads". In [Hau14b] we observed that bimodules can be regarded as algebras for the generalized non-symmetric $\infty$-operad $\Delta_{/[1]}^{\mathrm{op}} \rightarrow \Delta^{\mathrm{op}}$. Here is the obvious "many-object" version of this:

Definition 4.1. Given spaces $X$ and $Y$, we let $\Delta_{X, Y}^{\mathrm{op}} \rightarrow \triangle_{/[1]}^{\mathrm{op}}$ be a left fibration associated to the functor $\mathbb{\Delta}_{/[1]}^{\mathrm{p}} \rightarrow \mathcal{S}$ obtained as a right Kan extension of the functor $\{(0),(1)\} \rightarrow \mathcal{S}$ sending (0) to $X$ and (1) to $Y$.

The composite functor $\Delta_{X, Y}^{\mathrm{op}} \rightarrow \Delta_{/[1]}^{\mathrm{op}} \rightarrow \Delta^{\mathrm{op}}$ is a double $\infty$-category - this is a special case of Lemma 6.2, which we'll prove below.

Remark 4.2. An object of $\triangle_{/[1]}^{\mathrm{op}}$ can be described as a list $\left(i_{0}, \ldots, i_{n}\right)$ where $0 \leqslant i_{k} \leqslant$ $i_{k+1} \leqslant 1$. An object of $\triangle_{X_{0}, X_{1}}^{\mathrm{op}}$ lying over this is then a list $\left(x_{0}, \ldots, x_{n}\right)$ with $x_{k} \in X_{i_{k}}$. There are inclusions $\triangle_{X_{i}}^{\mathrm{op}} \hookrightarrow \triangle_{X_{0}, X_{1}}^{\mathrm{op}}$ lying over the two inclusions $\triangle^{\mathrm{op}} \rightarrow \triangle_{/[1]}^{\mathrm{op}}$ (given

${ }^{4}$ We do not recall the definition of these here, as we will not make use of this class of morphisms in this paper. 
by composing with the two maps $[0] \rightarrow[1]$ in $\Delta$ ). Suppose $\mathcal{V}$ is a monoidal $\infty$-category and $M: \triangle_{X_{0}, X_{1}}^{\mathrm{op}} \rightarrow \mathcal{V}^{\otimes}$ is a $\triangle_{X_{0}, X_{1}}^{\mathrm{op}}$-algebra in $\mathcal{V}$. If we write $\mathcal{C}$ and $\mathcal{D}$ for the two enriched $\infty$-categories obtained by restricting $M$ to $\triangle_{X_{0}}^{\mathrm{op}}$ and $\triangle_{X_{1}}^{\mathrm{op}}$, the additional data determined by $M$ can be described as:

- for $c \in X_{0}$ and $d \in X_{1}$, an object $M(c, d) \in \mathcal{V}$,

- for $c^{\prime}, c \in X_{0}$ and $d \in X_{1}$, a morphism $\mathcal{C}\left(c^{\prime}, c\right) \otimes M(c, d) \rightarrow M\left(c^{\prime}, d\right)$, coming from the map $\left(c^{\prime}, c, d\right) \rightarrow\left(c^{\prime}, d\right)$ (over $d_{1}:(0,0,1) \rightarrow(0,1)$ ),

- for $c \in X_{0}$ and $d, d^{\prime} \in X_{1}$, a morphism $M(c, d) \otimes \mathcal{D}\left(d, d^{\prime}\right) \rightarrow M\left(c, d^{\prime}\right)$, coming from the map $\left(c, d, d^{\prime}\right) \rightarrow\left(c, d^{\prime}\right)$ (over $d_{1}:(0,1,1) \rightarrow(0,1)$ ),

- for $c^{\prime}, c \in X_{0}$ and $d, d^{\prime} \in X_{1}$, a homotopy-commutative square

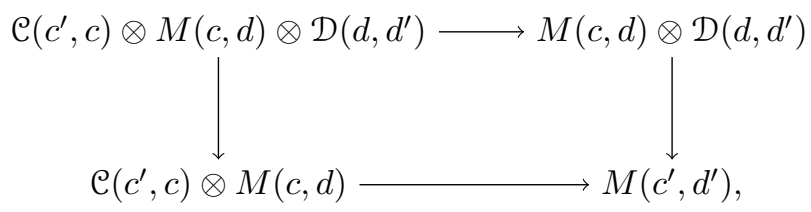

since the two maps $\left(c^{\prime}, c, d, d^{\prime}\right) \rightarrow\left(c^{\prime}, d^{\prime}\right)$ are homotopic,

- together with data showing that these action maps are homotopy-coherently compatible with the composition and unit maps in $\mathcal{C}$ and $\mathcal{D}$.

In other words, $M$ is precisely a homotopy-coherent version of the notion of bimodule for enriched categories we considered above.

Definition 4.3. The generalized non-symmetric $\infty$-operads $\triangle_{X, Y}^{\mathrm{op}}$ are clearly natural in $X$ and $Y$, so we get a functor $\mathcal{S}^{\times 2} \rightarrow \mathrm{Opd}_{\infty}^{\text {ns,gen }}$. If $\mathcal{V}$ is a monoidal $\infty$-category, we let $\operatorname{Bimod}_{\text {cat }}(\mathcal{V}) \rightarrow \mathcal{S}^{\times 2}$ be a Cartesian fibration associated to the functor sending $(X, Y)$ to $\operatorname{Alg}_{\triangle_{X, Y}^{\mathrm{op}}}(\mathcal{V})$. There are natural maps of generalized non-symmetric $\infty$ operads $\triangle_{X}^{\mathrm{op}}, \triangle_{Y}^{\mathrm{Op}} \hookrightarrow \triangle_{X, Y}^{\mathrm{op}}$, which leads to a functor $\operatorname{Bimod}_{\text {cat }}(\mathcal{V}) \rightarrow \operatorname{Alg}_{\text {cat }}(\mathcal{V})^{\times 2}$. If $\mathcal{C}$ and $\mathcal{D}$ are $\mathcal{V}$ - $\infty$-categories, we call an object of the fibre of this map at $(\mathcal{C}, \mathcal{D})$ a C-D-bimodule.

\section{Composing bimodules}

Let $\mathbf{V}$ be an ordinary monoidal category. If $\mathbf{A}, \mathbf{B}$, and $\mathbf{C}$ are $\mathbf{V}$-categories and we are given an A-B-bimodule $M$ and a $\mathbf{B}-\mathbf{C}$-bimodule $N$, their composite, which we'll denote $M \otimes_{\mathbf{B}} N$, is the $\mathbf{A}$-C-bimodule given by sending $(a, c)$ to the coequalizer

$$
\coprod_{b, b^{\prime} \in \mathbf{B}} M(a, b) \otimes \mathbf{B}\left(b, b^{\prime}\right) \otimes N\left(b^{\prime}, c\right) \rightrightarrows \coprod_{b \in \mathbf{B}} M(a, b) \otimes N(b, c),
$$

with the two maps given by the action of $\mathbf{B}$ on $M$ and $N$. In fact, this is a reflexive coequalizer, since we get a map in the other direction using the unit maps of $\mathbf{B}$. When passing from ordinary categories to $\infty$-categories the natural replacement of a reflexive coequalizer is usually the geometric realization of a simplicial object, and 
indeed there is a natural simplicial object extending this coequalizer diagram, namely:

$$
\begin{gathered}
\coprod_{b, b^{\prime}, b^{\prime \prime}, b^{\prime \prime \prime} \in \mathbf{B}} M(a, b) \otimes \mathbf{B}\left(b, b^{\prime}\right) \otimes \mathbf{B}\left(b^{\prime}, b^{\prime \prime}\right) \otimes \mathbf{B}\left(b^{\prime \prime}, b^{\prime \prime \prime}\right) \otimes N\left(b^{\prime \prime \prime}, c\right) \\
\coprod_{b, b^{\prime}, b^{\prime \prime} \in \mathbf{B}} M(a, b) \otimes \mathbf{B}\left(b, b^{\prime}\right) \otimes \mathbf{B}\left(b^{\prime}, b^{\prime \prime}\right) \otimes N\left(b^{\prime \prime}, c\right) \\
\coprod_{b, b^{\prime} \in \mathbf{B}} M(a, b) \otimes \mathbf{B}\left(\uparrow, \mid b^{\prime}\right) \otimes N\left(b^{\prime}, c\right) \\
\coprod_{b \in \mathbf{B}} M(a, b) \otimes N(b, c),
\end{gathered}
$$

where the face maps are given by the action of $\mathbf{B}$ on the bimodules, and the degeneracy maps by the unit maps for $\mathbf{B}$. We should therefore expect the composition of bimodules for enriched $\infty$-categories to be given by the colimit of a simplicial object analogous to this.

On the other hand, in [Hau14b] we defined the tensor product of bimodules for associative algebras as an operadic left Kan extension. This procedure has a natural generalization to the many-object setting, which gives a precise definition of the composite of two bimodules. We'll now introduce this, and then show that this operadic Kan extension is in fact given by taking the expected analogue of the colimit above.

Definition 5.1. Given spaces $X_{0}, X_{1}, X_{2}$, we let $\triangle_{X_{0}, X_{1}, X_{2}}^{\text {op }} \rightarrow \triangle_{/[2]}^{\text {op }}$ be the left fibration associated to the functor $\triangle_{/[2]}^{\mathrm{op}} \rightarrow \mathcal{S}$ obtained by right Kan extension from the functor $\{(0),(1),(2)\} \rightarrow \mathcal{S}$ sending $(i)$ to $X_{i}$.

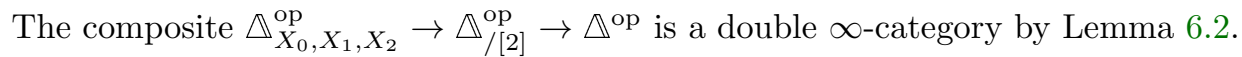

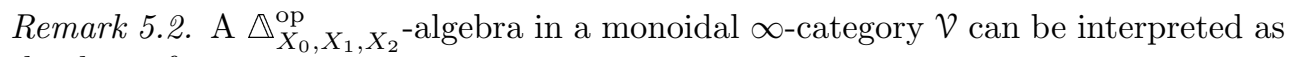
the data of:

- three $\mathcal{V}$ - $\infty$-categories $\mathfrak{C}_{i}$ with $X_{i}$ as space of objects $(i=0,1,2)$,

- three bimodules: for $0 \leqslant i<j \leqslant 2$, a $\mathcal{C}_{i}$ - $\mathrm{C}_{j}$-bimodule $M_{i j}$,

- a $\mathcal{C}_{1}$-bilinear map $M_{01} \otimes M_{12} \rightarrow M_{02}$, i.e. given $x_{i} \in X_{i}$ we have maps

$$
M_{01}\left(x_{0}, x_{1}\right) \otimes M_{12}\left(x_{1}, x_{2}\right) \rightarrow M_{02}\left(x_{0}, x_{2}\right),
$$

compatible with the action of $\mathcal{C}_{1}$.

We want to restrict ourselves to the case where this map exhibits $M_{02}$ as the tensor product or composite $M_{01} \otimes \mathfrak{e}_{1} M_{12}$. As in [Hau14b], we do this by considering only those $\triangle_{X_{0}, X_{1}, X_{2}}^{\mathrm{pp}}$ that arise as the left operadic Kan extensions of algebras for a subcategory of $\triangle_{X_{0}, X_{1}, X_{2}}^{\text {op }}$ :

Definition 5.3. Recall that a map $\phi:[n] \rightarrow[m]$ in $\Delta$ is said to be cellular if $\phi(i+$ $1)-\phi(i) \leqslant 1$ for all $i$, and that we write $\bigwedge_{/[n]}^{\mathrm{op}}$ for the full subcategory of $\triangle_{/[n]}^{\mathrm{op}}$ spanned 
by the cellular maps; this is a generalized non-symmetric $\infty$-operad by [Hau14b, Lemma 5.5]. We define $\mathbb{N}_{X_{0}, X_{1}, X_{2}}^{\mathrm{op}}$ by the pullback square

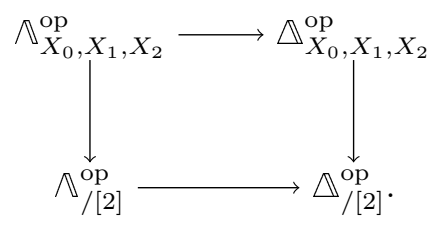

This is a pullback square in generalized non-symmetric $\infty$-operads, so $\mathbb{N}_{X_{0}, X_{1}, X_{2}}^{\mathrm{p}}$ is a generalized non-symmetric $\infty$-operad. Moreover, the inclusion

$$
\tau_{X_{0}, X_{1}, X_{2}}: \bigwedge_{X_{0}, X_{1}, X_{2}}^{\mathrm{op}} \rightarrow \triangle_{X_{0}, X_{1}, X_{2}}^{\mathrm{op}}
$$

is extendable in the sense of Definition 2.5 by Proposition 6.8 (i.e. operadic left Kan extensions along this map can be described using generalized non-symmetric

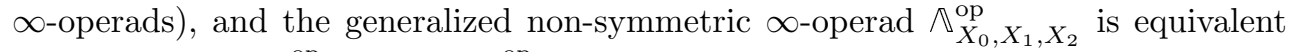
to the pushout $\triangle_{X_{0}, X_{1}}^{\mathrm{op}} \amalg_{\triangle_{X_{1}}^{\mathrm{op}}} \triangle_{X_{1}, X_{2}}^{\mathrm{op}}$ of generalized non-symmetric $\infty$-operads by Corollary 6.19.

This implies that if $\mathcal{V}$ is a monoidal $\infty$-category compatible with small colimits, the restriction

$$
\begin{aligned}
\tau_{X_{0}, X_{1}, X_{2}}^{*}: \operatorname{Alg}_{\triangle_{X_{0}, X_{1}, X_{2}}^{\text {op }}}(\mathcal{V}) & \rightarrow \operatorname{Alg}_{\triangle_{X_{0}, X_{1}, X_{2}}^{\text {op }}}(\mathcal{V}) \\
& \simeq \operatorname{Alg}_{\triangle_{X_{0}, X_{1}}^{\text {op }}}(\mathcal{V}) \times_{\operatorname{Alg}_{\triangle_{X_{1}}^{\text {op }}}(\mathcal{V})} \operatorname{Alg}_{\triangle_{X_{1}, X_{2}}^{\text {op }}}(\mathcal{V})
\end{aligned}
$$

has a fully faithful left adjoint $\tau_{X_{0}, X_{1}, X_{2}, !}$ for all spaces $X_{0}, X_{1}, X_{2}$. If $\mathcal{C}_{i}$ is a $\mathcal{V}$ - $\infty$-category with space of objects $X_{i}$ for $i=0,1,2$, and we have a $\mathfrak{C}_{0}-\mathcal{C}_{1}$-bimodule $M$ and a $\mathfrak{C}_{1}-\mathcal{C}_{2}$-bimodule $N$, the composite $\mathcal{C}_{0}-\mathfrak{C}_{2}$-bimodule $M \otimes_{\mathfrak{C}_{1}} N$ is the restriction to $\triangle_{X_{0}, X_{2}}^{\mathrm{op}}$ of the $\triangle_{X_{0}, X_{1}, X_{2}}^{\mathrm{op}}$-algebra obtained by applying $\tau_{X_{0}, X_{1}, X_{2}, !}$ to the ${\mathbb{N}_{X_{0}, X_{1}, X_{2}}^{\mathrm{op}}}^{-}$ algebra corresponding to $M$ and $N$.

Remark 5.4. Let $\operatorname{Bimod}_{\text {cat }}^{2}(\mathcal{V}) \rightarrow \mathcal{S}^{\times 3}$ be the Cartesian fibration associated to the functor $\left(\mathcal{S}^{\times 3}\right)^{\mathrm{op}} \rightarrow \mathrm{Cat}_{\infty}$ that sends $(X, Y, Z)$ to $\operatorname{Alg}_{\triangle_{X, Y, Z}^{\mathrm{op}}}(\mathcal{V})$. The left adjoints

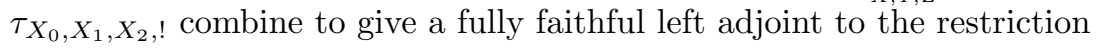

$$
\operatorname{Bimod}_{\text {cat }}^{2}(\mathcal{V}) \rightarrow \operatorname{Bimod}_{\text {cat }}(\mathcal{V}) \times \operatorname{Alg}_{\text {cat }}(\mathcal{V}) \operatorname{Bimod}_{\text {cat }}(\mathcal{V})
$$

Combining this with the appropriate projection $\operatorname{Bimod}_{\text {cat }}^{2}(\mathcal{V}) \rightarrow \operatorname{Bimod}_{\text {cat }}(\mathcal{V})$ we get a composition functor

$$
\operatorname{Bimod}_{\text {cat }}(\mathcal{V}) \times \operatorname{Alg}_{\text {cat }}(\mathcal{V}) \operatorname{Bimod}_{\text {cat }}(\mathcal{V}) \rightarrow \operatorname{Bimod}_{\text {cat }}(\mathcal{V}) .
$$

Now we want to see that this composition of bimodules is given by forming the expected colimit. The key observation is the following:

Proposition 5.5. Given spaces $X, Y, Z$ and points $x \in X$ and $z \in Z$, let $X_{x, z}$ be the $\infty$-category determined by the pullback square

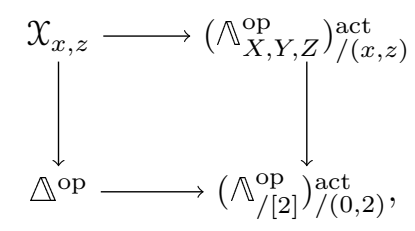


where the bottom horizontal map sends $[n]$ to the object $(0,1, \ldots, 1,2)$ with $n+1$ copies of 1 . Then:

(i) There is an equivalence $x_{x, z} \stackrel{\sim}{\rightarrow} \triangle_{Y}^{\text {op }}$.

(ii) The top horizontal map $\mathcal{X}_{x, z} \rightarrow\left(\wedge_{X, Y, Z}^{\mathrm{op}}\right)_{/(x, z)}^{\mathrm{act}}$ is cofinal.

Proof. The projection $\left(\bigwedge_{X, Y, Z}^{\mathrm{op}}\right)_{/(x, z)}^{\mathrm{act}} \rightarrow\left(\mathbb{\Lambda}_{/[2]}^{\mathrm{op}}\right)_{/(0,2)}^{\text {act }}$ is a left fibration by Lemma $6.7(\mathrm{i})$, hence so is the pullback $x_{x, z} \rightarrow \triangle^{\mathrm{op}}$. The functor taking a space $S$ to the left fibration $\triangle_{S}^{\mathrm{op}} \rightarrow \triangle^{\mathrm{op}}$ is right adjoint to the functor taking a left fibration over $\mathbb{\Delta}^{\mathrm{op}}$ to its fibre at [0]. Since the fibre of $X_{x, y}$ at $[n]$ is $X_{/ x} \times Y^{\times(n+1)} \times Z_{/ z} \simeq Y^{\times(n+1)}$, the unit of this adjunction gives a map of left fibrations $X_{x, z} \rightarrow \triangle_{Y}^{\mathrm{op}}$. To see that this is an equivalence it suffices to show that the induced map on fibres at any $[n] \in \mathbb{\Delta}^{\text {op }}$ is an equivalence, which is clear. This proves (i).

The bottom horizontal map $\Delta^{\mathrm{op}} \rightarrow\left(\mathbb{\Lambda}_{/[2]}^{\mathrm{op}}\right)_{/(0,2)}^{\text {act }}$ is cofinal by [Hau14b, Lemma 4.17]. Combining [Lur09, Proposition 4.1.2.15] with [Lur09, Remark 4.1.2.10] we know that the pullback of a cofinal map along a coCartesian fibration is cofinal, which proves (ii).

From the definition of left operadic Kan extensions it therefore follows that if $\mathcal{A}$, $\mathcal{B}$, and $\mathcal{C}$ are $\mathcal{V}$ - $\infty$-categories with spaces of objects $X, Y$, and $Z$, respectively, and we have an $\mathcal{A}$ - $\mathcal{B}$-bimodule $M$ and a $\mathcal{B}$ - $\mathcal{C}$-bimodule $N$, then the composite $\mathcal{A}$-C-bimodule $M \otimes_{\mathcal{B}} N$ is given at $(x, z)$ by a $\triangle_{Y}^{\text {op }}$-indexed colimit we can informally write as

$$
M \otimes_{\mathcal{B}} N \simeq \operatorname{colim}_{\left(y_{0}, \ldots, y_{n}\right) \in \triangle_{Y}^{\text {op }}} M\left(x, y_{0}\right) \otimes \mathcal{B}\left(y_{0}, y_{1}\right) \otimes \cdots \otimes \mathcal{B}\left(y_{n-1}, y_{n}\right) \otimes N\left(y_{n}, z\right) .
$$

To relate this to the expected geometric realization, we need a technical observation:

Proposition 5.6. Let $\mathcal{J}$ be an $\infty$-category and $p: \mathcal{J} \rightarrow \mathrm{Cat}_{\infty}$ a functor with $\mathcal{K} \rightarrow \mathcal{J}$ its associated coCartesian fibration. Suppose $q: \mathcal{K} \rightarrow \mathcal{D}$ is a functor such that for each $\alpha \in \mathcal{J}$ the diagram $q_{\alpha}: p(\alpha) \simeq \mathcal{K}_{\alpha} \rightarrow \mathcal{D}$ has a colimit; by [Lur09, Proposition 4.2.2.7] there exists an (essentially unique) map $q_{+}: \mathcal{K}_{+} \rightarrow \mathcal{D}$, where

$$
\mathcal{K}_{+}:=\mathcal{K} \times \Delta^{1} \amalg_{\mathcal{K} \times\{1\}} \mathcal{J},
$$

that restricts to $q$ on $\mathcal{K}$ and to a colimit of $q_{\alpha}$ on $p(\alpha)^{\triangleright} \simeq \mathcal{K}_{+} \times_{\mathcal{J}}\{\alpha\}$. Then the maps

$$
\mathcal{D}_{q /} \leftarrow \mathcal{D}_{q_{+} /} \rightarrow \mathcal{D}_{\left.q_{+}\right|_{\mathcal{J}} /}
$$

are trivial fibrations.

Proof. The map $\mathcal{K} \times\{1\} \hookrightarrow \mathcal{K} \times \Delta^{1}$ is right anodyne by [Lur09, Corollary 2.1.2.7], so the pushout $\mathcal{J} \rightarrow \mathcal{K}_{+}$is also right anodyne and thus cofinal by [Lur09, Proposition 4.1.1.3]. Therefore $\mathcal{D}_{q_{+} /} \rightarrow \mathcal{D}_{\left.q_{+}\right|_{\jmath} /}$ is a trivial fibration by [Lur09, Proposition 4.1.1.7]. On the other hand, since $\pi: \mathcal{K} \rightarrow \mathcal{J}$ is a coCartesian fibration, for each $i \in \mathcal{J}$ the inclusion

$$
\mathcal{K}_{i} \hookrightarrow \mathcal{K}_{/ i}:=\mathcal{K}_{\times_{\mathcal{J}}} \mathcal{J}_{/ i}
$$

is cofinal - this follows by applying [Lur09, Theorem 4.1.3.1], since for every object $(k, f: \pi(k) \rightarrow i) \in \mathcal{K}_{/ i}$ the fibre $\left(\mathcal{K}_{i}\right)_{(k, f) /}$ has an initial object given by the coCartesian map $k \rightarrow f_{!} k$ and so is weakly contractible. Thus $q_{+}$is a left Kan extension of $q$ along $\mathcal{K} \hookrightarrow \mathcal{K}_{+}$, and hence $\mathcal{D}_{q_{+} /} \rightarrow \mathcal{D}_{q /}$ is a trivial fibration by [Lur09, Lemma 4.3.2.7]. 
Corollary 5.7. Let $q: \mathcal{K} \rightarrow \mathcal{D}$ be as above. If the diagram $q$ has a colimit, we have an equivalence

$$
\underset{\mathcal{K}}{\operatorname{colim}} q \simeq \operatorname{colim}_{\alpha \in \mathcal{J}} \operatorname{colim}_{p(\alpha)} q_{\alpha}
$$

where the functoriality in $\alpha$ of the colimits over $p(\alpha)$ comes from the diagram $q_{+}$.

Proof. Since the maps in Proposition 5.6 are trivial fibrations compatible with the projections to $\mathcal{D}$, the colimit of $q$, which is the initial object of $\mathcal{D}_{q /}$, must project to an object of $\mathcal{D}$ that is equivalent to the projection of the initial object of $\mathcal{D}_{\left.q_{+}\right|_{\mathcal{J}} /}$, which is a colimit of the diagram $\alpha \mapsto \operatorname{colim}_{p(\alpha)} q_{\alpha}$ induced by $q_{+}$.

Since $\triangle_{Y}^{\text {op }} \rightarrow \triangle^{\text {op }}$ is a left fibration, we can apply this to our $\triangle_{Y}^{\text {op }}$-indexed colimit for $\left(M \otimes_{\mathcal{B}} N\right)(x, z)$ to conclude that, as we expected, this is equivalent to the geometric realization of a simplicial diagram with $n$th term

$$
\operatorname{colim}_{\left(y_{0}, \ldots, y_{n}\right) \in Y^{\times(n+1)}} M\left(a, y_{0}\right) \otimes \mathcal{B}\left(y_{0}, y_{1}\right) \otimes \cdots \otimes \mathcal{B}\left(y_{n-1}, y_{n}\right) \otimes N\left(y_{n}, c\right) .
$$

\section{The double $\infty$-category of enriched $\infty$-categories}

Now we get to the meat of this paper - in this section we'll construct a double $\infty$-category of $\mathcal{V}$ - $\infty$-categories, in the form of a simplicial $\infty$-category whose value at $[0]$ is $\operatorname{Alg}_{\text {cat }}(\mathcal{V})$ and at [1] is $\operatorname{Bimod}_{\text {cat }}(\mathcal{V})$, with the composition

$$
\operatorname{Bimod}_{\text {cat }}(\mathcal{V}) \times_{\operatorname{Alg}_{\text {cat }}(\mathcal{V})} \operatorname{Bimod}_{\text {cat }}(\mathcal{V}) \rightarrow \operatorname{Bimod}_{\text {cat }}(\mathcal{V})
$$

given by the construction we discussed in the previous section.

The basic objects we consider are again the natural many-object versions of those we used in [Hau14b]:

Definition 6.1. Given spaces $X_{0}, \ldots, X_{n}$ we define $\Delta_{X_{0}, \ldots, X_{n}}^{\mathrm{op}} \rightarrow \triangle_{/[n]}^{\mathrm{op}}$ to be the coCartesian fibration associated to the functor $\mathbb{\Delta}_{/[n]}^{\mathrm{op}} \rightarrow \mathcal{S}$ obtained by right Kan extension from the functor $\{(0), \ldots,(n)\} \rightarrow \mathcal{S}$ that sends $(i)$ to $X_{i}$.

Lemma 6.2. The composite $\Delta_{X_{0}, \ldots, X_{n}}^{\mathrm{op}} \rightarrow \triangle_{/[n]}^{\mathrm{op}} \rightarrow \Delta^{\mathrm{op}}$ is a double $\infty$-category for all spaces $X_{0}, \ldots, X_{n}$.

Proof. The right Kan-extended functor $F: \mathbb{\Delta}_{/[n]}^{\mathrm{op}} \rightarrow \mathcal{S}$ clearly sends $\left(i_{0}, \ldots, i_{n}\right)$ to $X_{i_{0}} \times \cdots \times X_{i_{n}}$, and so is a $\triangle_{/[n]}^{\text {op }}$-category object in the sense of [GH15, Definition 3.5.2], i.e. it satisfies the Segal condition

$$
F\left(i_{0}, \ldots, i_{n}\right) \stackrel{\sim}{\rightarrow} F\left(i_{0}, i_{1}\right) \times_{F\left(i_{1}\right)} \cdots \times_{F\left(i_{n-1}\right)} F\left(i_{n-1}, i_{n}\right) .
$$

The composite coCartesian fibration $\triangle_{X_{0}, \ldots, X_{n}}^{\mathrm{op}} \rightarrow \triangle^{\mathrm{op}}$ is therefore a double $\infty$-category by [GH15, Proposition 3.5.4].

Definition 6.3. If $\mathcal{C}$ is an $\infty$-category, let $\Delta_{e} \rightarrow \Delta$ be a Cartesian fibration associated to the functor $\Delta^{\mathrm{op}} \rightarrow$ Cat $_{\infty}$ that is the right Kan extension of the functor $\{[0]\} \rightarrow \mathrm{Cat}_{\infty}$ sending $[0]$ to $\mathcal{C}$. (This functor sends $[n]$ to $\mathrm{C}^{\times(n+1)}$.)

We now observe that the double $\infty$-categories $\triangle_{X_{0}, \ldots, X_{n}}^{\text {op }}$ combine to a functor $\Delta_{S} \rightarrow \mathrm{Opd}_{\infty}^{\mathrm{ns}, \mathrm{gen}}:$ 
Definition 6.4. Let $i_{n}:\{0, \ldots, n\} \hookrightarrow \mathbb{\Delta}_{/[n]}^{\mathrm{op}}$ be the functor that takes $j \in\{0, \ldots, n\}$ to the map $[0] \rightarrow[n]$ sending 0 to $j \in[n]$. (This is equivalent to the inclusion of the fibre of $\triangle_{/[n]}^{\mathrm{op}}$ over $[0] \in \triangle^{\mathrm{op}}$.) Right Kan extension along $i_{n}$ then determines a functor

$$
\mathcal{S}^{\times(n+1)} \simeq \operatorname{Fun}(\{0, \ldots, n\}, \mathcal{S}) \rightarrow \operatorname{Fun}\left(\triangle_{/[n]}^{\mathrm{op}}, \mathcal{S}\right),
$$

which is moreover natural in $[n] \in \triangle^{\mathrm{op}}$. Using the equivalence between functors to $\mathcal{S}$ and left fibrations, we get a functor from $\mathcal{S}^{\times(n+1)}$ to left fibrations over $\triangle_{/[n]}^{\mathrm{op}}$ that takes $\left(X_{0}, \ldots, X_{n}\right)$ to $\triangle_{X_{0}, \ldots, X_{n}}^{\mathrm{op}} \rightarrow \triangle_{/[n]}^{\mathrm{op}}$. Since the resulting composite maps $\triangle_{X_{0}, \ldots, X_{n}}^{\mathrm{op}} \rightarrow \triangle^{\mathrm{op}}$ are double $\infty$-categories, we get functors $\mathcal{S}^{\times(n+1)} \rightarrow \mathrm{Opd}_{\infty}^{\mathrm{ns}, \mathrm{gen}}$ taking $\left(X_{0}, \ldots, X_{n}\right)$ to $\triangle_{X_{0}, \ldots, X_{n}}^{\text {op }} \rightarrow \triangle^{\mathrm{op}}$, natural in $n$. Using the universal property of $\Delta_{\mathcal{S}}$ from [GHN15, Proposition 7.3] this corresponds to a functor $\Delta_{\mathcal{S}} \rightarrow \operatorname{Opd}_{\infty}^{\mathrm{ns}, \mathrm{gen}}$.

Definition 6.5. For any generalized non-symmetric $\infty$-operad $\mathcal{O}$, we let

$$
{\overline{\mathfrak{A L} \mathfrak{G}_{\text {cat }}^{\prime}}}^{\prime}(\mathcal{O}) \rightarrow \triangle_{\mathcal{S}}
$$

be a Cartesian fibration associated to the functor $\left(\triangle_{\mathcal{S}}\right)^{\mathrm{op}} \rightarrow$ Cat ${ }_{\infty}$ given by

$$
\left(X_{0}, \ldots, X_{n}\right) \mapsto \operatorname{Alg}_{\triangle_{X_{0}, \ldots, X_{n}}^{\text {op }}}(\mathcal{O}) .
$$

Then we define $\overline{\mathfrak{A L G}}_{\text {cat }}(\mathcal{O}) \rightarrow \triangle^{\text {op }}$ to be a coCartesian fibration corresponding to the composite Cartesian fibration $\overline{\mathfrak{A L G}}_{\text {cat }}^{\prime}(\mathcal{O}) \rightarrow \Delta_{S} \rightarrow \Delta$. In particular, over $[n] \in \triangle^{\text {op }}$ we have a Cartesian fibration $\overline{\mathfrak{A L G}}_{\text {cat }}(\mathcal{O})_{[n]} \rightarrow \mathcal{S}^{\times(n+1)}$.

Definition 6.6. For spaces $X_{0}, \ldots, X_{n}$, we define a generalized non-symmetric $\infty$ operad $\wedge_{X_{0}, \ldots, X_{n}}^{\mathrm{pp}}$ by the pullback diagram

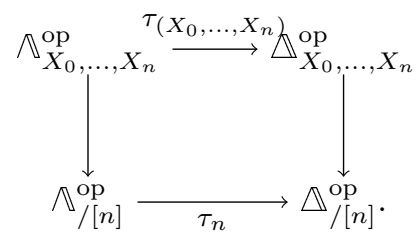

We say that a $\triangle_{X_{0}, \ldots, X_{n}}^{\mathrm{op}}$-algebra is composite if it is the operadic left Kan extension

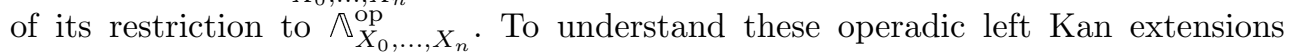
we must check that the map $\bigwedge_{X_{0}, \ldots, X_{n}}^{\mathrm{op}} \rightarrow \triangle_{X_{0}, \ldots, X_{n}}^{\mathrm{op}}$ is extendable, in the sense of Definition 2.5.

To prove this we first make the following technical observation:

Lemma 6.7. Suppose given spaces $X_{0}, \ldots, X_{n}$, a morphism $\xi:[m] \rightarrow[n]$ in $\triangle$, and $\Xi \in \triangle_{X_{0}, \ldots, X_{n}}^{\mathrm{op}}$ over $\xi \in \mathbb{\Delta}_{/[n]}^{\mathrm{op}}$. Then:

(i) The projection

$$
\left(\bigwedge_{X_{0}, \ldots, X_{n}}^{\mathrm{op}}\right)_{/ \Xi}^{\mathrm{act}} \rightarrow\left(\mathbb{\Lambda}_{/[n]}^{\mathrm{op}}\right)_{/ \xi}^{\mathrm{act}}
$$

is a left fibration.

(ii) For any cellular morphism $\eta:[k] \rightarrow[n]$ and active morphism $\phi:[m] \rightarrow[k]$ in $\Delta$ such that $\xi=\eta \phi$, the fibre of this projection at $(\phi, \eta)$ is the pullback 


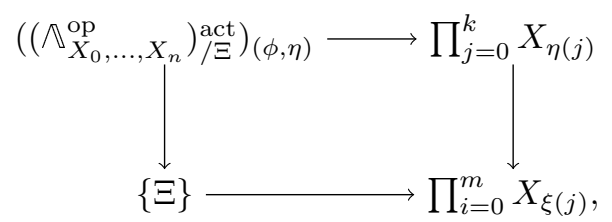

where the right vertical map sends $\left(p_{0}, \ldots, p_{m}\right)$ to $\left(p_{\phi(0)}, \ldots, p_{\phi(m)}\right)$.

Proof. First consider the commutative diagram

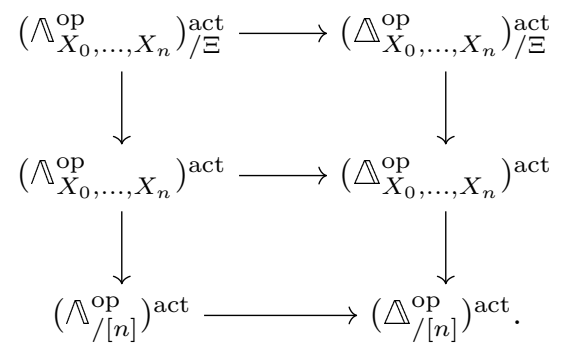

Here the top square is Cartesian by the definition of $\left(\wedge_{X_{0}, \ldots, X_{n}}^{\mathrm{op}}\right)_{/ \Xi}^{\text {act }}$, and it follows

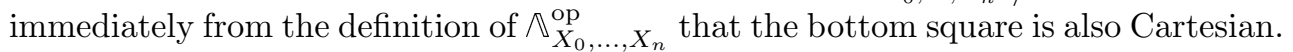
Thus the composite square is also Cartesian.

Now consider the diagram

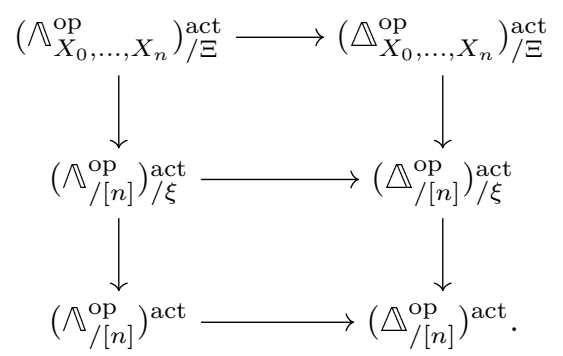

Here the bottom square is Cartesian by the definition of $\left(\mathcal{N}_{/[n]}^{\mathrm{op}}\right)_{/ \xi}^{\text {act }}$, hence since the composite square is Cartesian so is the top square.

The projection $\triangle_{X_{0}, \ldots, X_{n}}^{\mathrm{op}} \rightarrow \triangle_{/[n]}^{\mathrm{op}}$ is by definition a left fibration, hence so is the restriction $\left(\triangle_{X_{0}, \ldots, X_{n}}^{\mathrm{op}}\right)^{\text {act }} \rightarrow\left(\triangle_{/[n]}^{\mathrm{op}}\right)^{\text {act }}$ to the active maps, since this can be described as the pullback along $\triangle_{\text {act }}^{\text {op }} \rightarrow \triangle^{\text {op }}$. The projection $\left(\triangle_{X_{0}, \ldots, X_{n}}^{\text {op }}\right)_{/ \Xi}^{\text {act }} \rightarrow\left(\triangle^{n, \text { op }}\right)_{/ \xi}^{\text {act }}$ is therefore a left fibration by [Lur09, Proposition 2.1.2.1], hence so is the pullback $\left(\wedge_{X_{0}, \ldots, X_{n}}^{\mathrm{op}}\right)_{/ \Xi}^{\text {act }} \rightarrow\left(\wedge_{/[n]}^{\mathrm{op}}\right)_{/ \xi}^{\text {act }}$. This proves $(\mathrm{i})$.

(ii) then follows immediately from the definition of the left fibration $\Delta_{X_{0}, \ldots, X_{n}}^{\mathrm{op}} \rightarrow$ $\triangle_{/[n]}^{\mathrm{op}}$.

Proposition 6.8. For any spaces $X_{0}, \ldots, X_{n}$, the inclusion $\bigwedge_{X_{0}, \ldots, X_{n}}^{\mathrm{op}} \rightarrow \triangle_{X_{0}, \ldots, X_{n}}^{\mathrm{op}}$ is extendable. 
Proof. We must show that for any $\Xi \in \triangle_{X_{0}, \ldots, X_{n}}^{\mathrm{op}}$ (lying over $\xi:[m] \rightarrow[n]$ ), the map

$$
\left(\wedge_{X_{0}, \ldots, X_{n}}^{\mathrm{op}}\right)_{/ \Xi}^{\mathrm{act}} \rightarrow \prod_{p=1}^{m}\left(\bigwedge_{X_{0}, \ldots, X_{n}}^{\mathrm{op}}\right)_{/ \rho_{p}^{*} \Xi}^{\mathrm{act}}
$$

is cofinal. Consider the commutative square

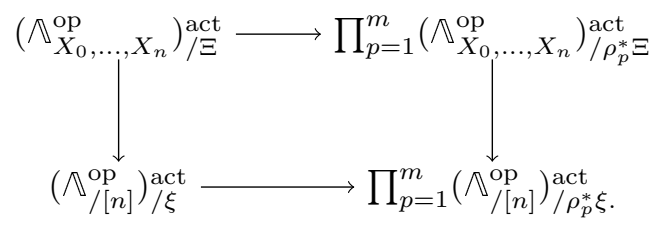

Here the vertical maps are left fibrations by Lemma 6.7(i), and the bottom horizontal map is cofinal by [Hau14b, Proposition 4.16]. [Lur09, Proposition 4.1.2.15] together with [Lur09, Remark 4.1.2.10] implies that the pullback of a cofinal map along a coCartesian fibration is cofinal, so to show that the top horizontal map is cofinal it's enough to prove that this square is Cartesian. Since the vertical maps are left fibrations, for this it suffices to show that the induced map on the fibres at any $(\phi, \eta) \in\left(\mathbb{\Lambda}_{/[n]}^{\mathrm{op}}\right)_{/ \xi}^{\text {act }}$ is an equivalence, which is clear from the description of the fibres in Lemma 6.7(ii).

Applying [Hau14b, Corollary A.60] (which itself is a minor variation of [Lur14, Theorem 3.1.2.3]), we get:

Corollary 6.9. Suppose $\mathcal{V}$ is a monoidal $\infty$-category compatible with small colimits. Then for any spaces $X_{0}, \ldots, X_{n}$ the restriction

$$
\tau_{X_{0}, \ldots, X_{n}}^{*}: \operatorname{Alg}_{\triangle_{X_{0}, \ldots, X_{n}}^{\text {op }}}(\mathcal{V}) \rightarrow \operatorname{Alg}_{\mathbb{N}_{X_{0}, \ldots, X_{n}}^{\text {op }}}(\mathcal{V})
$$

has a fully faithful left adjoint $\tau_{X_{0}, \ldots, X_{n}, !}$.

Definition 6.10. Suppose $\mathcal{V}$ is a monoidal $\infty$-category compatible with small colimits. We say a $\triangle_{X_{0}, \ldots, X_{n}}^{\text {op }}$-algebra $\mathcal{M}$ in $\mathcal{V}$ is composite if it lies in the image of $\tau_{X_{0}, \ldots, X_{n}, !}$, or equivalently if the counit map $\tau_{X_{0}, \ldots, X_{n}, !} \tau_{X_{0}, \ldots, X_{n}}^{*} \mathcal{M} \rightarrow \mathcal{M}$ is an equivalence.

Definition 6.11. Suppose $\mathcal{V}$ is a monoidal $\infty$-category compatible with small colimits. Let $\mathfrak{A} \mathfrak{L G}_{\text {cat }}(\mathcal{V})$ denote the full subcategory of $\overline{\mathfrak{A L}}_{\text {cat }}(\mathcal{V})$ spanned by the composite $\triangle_{X_{0}, \ldots, X_{n}}^{\mathrm{op}}$-algebras for all spaces $X_{0}, \ldots, X_{n}$.

Remark 6.12. The natural transformations $\bigwedge_{X_{0}, \ldots, X_{n}}^{\mathrm{op}} \rightarrow \triangle_{X_{0}, \ldots, X_{n}}^{\mathrm{op}}$ induce a map of Cartesian fibrations

$$
\tau_{n}^{*}:{\overline{\mathfrak{A} \mathfrak{L} \mathfrak{G}_{\text {cat }}}}(\mathcal{V})_{n} \rightarrow{\overline{\mathfrak{A} \mathfrak{L} \mathfrak{G}_{\text {cat }}}}^{\wedge}(\mathcal{V})_{n}
$$

over $\mathcal{S}^{\times(n+1)}$, where $\overline{\mathfrak{A L G}}_{\text {cat }}^{\wedge}(\mathcal{V})_{n} \rightarrow \mathcal{S}^{\times(n+1)}$ is a Cartesian fibration associated to the functor $\left(\mathcal{S}^{\times(n+1)}\right)^{\text {op }} \rightarrow$ Cat $_{\infty}$ that sends $\left(X_{0}, \ldots, X_{n}\right)$ to $\operatorname{Alg}_{\mathcal{N}_{X_{0}, \ldots, X_{n}}^{\text {op }}}(\mathcal{V})$. If $\mathcal{V}$ is compatible with small colimits, the fibrewise left adjoints $\tau_{X_{0}, \ldots, X_{n}, !}$ then combine to give a left adjoint $\tau_{n, !}: \overline{\mathfrak{A L G}}_{\text {cat }}^{\wedge}(\mathcal{V})_{n} \rightarrow \overline{\mathfrak{A L G}}_{\text {cat }}(\mathcal{V})_{n}$ by [Lur14, Proposition 7.3.2.6], and we can define $\mathfrak{A} \mathfrak{L} \mathfrak{G}_{\text {cat }}(\mathcal{V})_{n}$ to be the image of $\tau_{n, !}$. In particular, the projection $\mathfrak{A} \mathfrak{L} \mathfrak{G}_{\text {cat }}(\mathcal{V})_{[n]} \rightarrow \mathcal{S}^{\times(n+1)}$ is still a Cartesian fibration. 
Next we need to show that the projection $\mathfrak{A L G}_{\text {cat }}(\mathcal{V}) \rightarrow \mathbb{\Delta}^{\text {op }}$ is a coCartesian fibration. This will follow from an extension of [Hau14b, Proposition 4.37]:

Definition 6.13. Recall that for injective $\phi:[m] \rightarrow[n]$ in $\triangle$, we say that a morphism $\alpha:[k] \rightarrow[n]$ is $\phi$-cellular if

(1) for $i$ such that $\alpha(i)<\phi(0)$ we have $\alpha(i+1) \leqslant \alpha(i)+1$,

(2) for $i$ such that $\phi(j) \leqslant \alpha(i)<\phi(j+1)$ we have $\alpha(i+1) \leqslant \phi(j+1)$,

(3) for $i$ such that $\alpha(i) \geqslant \phi(m)$ we have $\alpha(i+1) \leqslant \alpha(i)+1$.

We write $\mathbb{N}_{/[n]}^{\mathrm{op}}[\phi]$ for the full subcategory of ${\triangle_{n}^{\mathrm{op}}}_{n}$ spanned by the $\phi$-cellular maps to $[n]$, and for spaces $X_{0}, \ldots, X_{n}$ we define $\wedge_{X_{0}, \ldots, X_{n}}^{\mathrm{op}}[\phi]$ by the pullback square

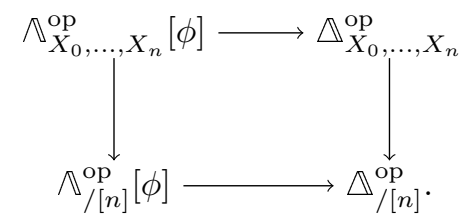

Proposition 6.14. (i) For any injective $\phi:[m] \rightarrow[n]$ and $\Gamma \in \triangle_{X_{\phi(0)}, \ldots, X_{\phi(m)}}^{\mathrm{op}}$ over $\phi:[k] \rightarrow[m] \in \mathbb{\triangle}_{/[m]}^{\mathrm{op}}$, the map

$$
\left(\wedge_{X_{\phi(0)}, \ldots, X_{\phi(m)}}^{\text {op }}\right)_{/ \Gamma}^{\text {act }} \rightarrow\left(\bigwedge_{X_{0}, \ldots, X_{n}}^{\text {op }}[\phi]\right)_{/ \phi_{*} \Gamma}^{\text {act }}
$$

is cofinal.

(ii) For any surjective $\phi:[m] \rightarrow[n]$ and $\Gamma \in \triangle_{X_{\phi(0)}, \ldots, X_{\phi(m)}}^{\mathrm{op}}$ over $\phi:[k] \rightarrow[m] \in \triangle_{/[m]}^{\mathrm{op}}$, the map

$$
\left(\bigwedge_{X_{\phi(0)}, \ldots, X_{\phi(m)}}^{\mathrm{op}}\right)_{/ \Gamma}^{\mathrm{act}} \rightarrow\left(\bigwedge_{X_{0}, \ldots, X_{n}}^{\mathrm{op}}\right)_{/ \phi_{*} \Gamma}^{\mathrm{act}}
$$

is cofinal.

Proof. Suppose $\phi:[m] \rightarrow[n]$ is injective. Consider the commutative square

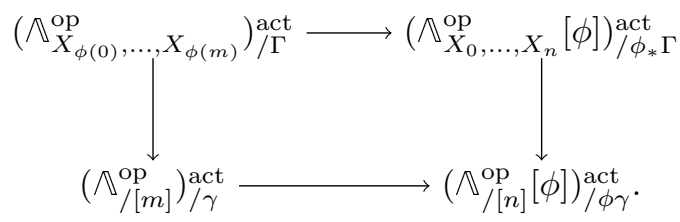

The proof of Lemma 6.7 clearly extends to the $\phi$-cellular case, so the vertical maps here are left fibrations and the bottom horizontal map is cofinal by [Hau14b, Proposition 4.37]. [Lur09, Proposition 4.1.2.15] together with [Lur09, Remark 4.1.2.10] implies that the pullback of a cofinal map along a coCartesian fibration is cofinal, so to show that the top horizontal map is cofinal it's enough to prove that this square is Cartesian. Since the vertical maps are left fibrations, for this it suffices to show that the induced map on the fibres at any object of $\left(\mathbb{N}_{/[m]}^{\mathrm{op}}\right)_{/ \gamma}^{\text {act }}$ is an equivalence, which is clear from the description of the fibres in Lemma 6.7(ii). This proves (i), and (ii) follows by the same argument.

Corollary 6.15. The restricted projection $\mathfrak{A L G}_{\text {cat }}(\mathcal{V}) \rightarrow \triangle^{\text {op }}$ is a coCartesian fibration. 
Proof. This follows, using Proposition 6.14, by exactly the same proof as that of [Hau14b, Corollary 4.38].

It follows that $\mathfrak{A L G}_{\text {cat }}(\mathcal{V}) \rightarrow \triangle^{\text {op }}$ determines a functor ${\Delta^{o p}}^{\text {op }} \rightarrow$ Cat $_{\infty}$. We want to show that $\mathfrak{A} \mathfrak{L} \mathfrak{G}_{\text {cat }}(\mathcal{V})$ is a double $\infty$-category, i.e. that this functor satisfies the Segal condition. We'll deduce this from the following observation:

Proposition 6.16. Let $\mathfrak{P}=\left(\mathcal{C}, S,\left\{K_{\alpha}^{\triangleleft} \rightarrow \mathcal{C}\right\}\right)$ be a categorical pattern, in the sense of [Lur14, B.0.19], and suppose $f: A \rightarrow B$ is a trivial cofibration in the model category $\left(\text { Set }_{\Delta}^{+}\right)_{\mathfrak{P}}$ of [Lur14, Theorem B.0.20] of one of the following kinds:

(a) $\left(\Lambda_{i}^{n}, T\right) \hookrightarrow\left(\Delta^{n}, T\right)$, where $T$ consists of the degenerate edges together with ( $i-$ 1) $\rightarrow i$, for all $0<i<n$.

(b) $\left(\partial \Delta^{n} \star K_{\alpha}, T\right) \hookrightarrow\left(\Delta^{n} \star K_{\alpha}, T\right)$, where $T$ consists of the non-degenerate edges together with all edges in $K_{\alpha}$ and all maps $n \rightarrow k$ for $k \in K_{\alpha}$.

Then for any pullback diagram

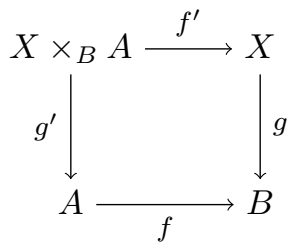

in $\left(\operatorname{Set}_{\Delta}^{+}\right)_{\mathfrak{P}}$ where $g$ is a fibration, the morphism $f^{\prime}: X \times_{B} A \rightarrow X$ is a weak equivalence.

Proof. In the case (a) this follows from [Lur14, Lemma 2.4.4.6] and in the case (b) it holds by the same argument as in the proofs of [Lur14, Lemmas 2.4.4.4 and 2.4.4.5].

Definition 6.17. As in [Hau14b, $\S 4.3]$, we write $\triangle_{/[n]}^{\amalg, o p}$ for the ordinary colimit $\triangle_{/[1]}^{\mathrm{op}} \amalg_{\triangle_{/[0]}^{\mathrm{op}}} \cdots \amalg_{\triangle_{[[0]}^{\mathrm{op}}}{\Delta_{/[1]}^{\mathrm{op}}}^{\mathrm{o}}$ in (marked) simplicial sets (over $\triangle^{\mathrm{op}}$ ), with $n$ copies of $\triangle_{/[1]}^{\mathrm{op}}$. For any Kan complexes $X_{0}, \ldots, X_{n}$, we then define $\triangle_{X_{0}, \ldots, X_{n}}^{\mathrm{L}, \mathrm{op}}$ by the (ordinary) pullback

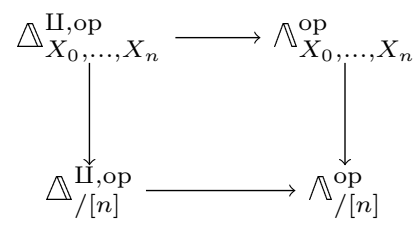

in $\left(\operatorname{Set}_{\Delta}^{+}\right)_{\mathfrak{O}}^{\mathrm{gen}}$.

Lemma 6.18. For any Kan complexes $X_{0}, \ldots, X_{n}$, the marked simplicial set $\triangle_{X_{0}, \ldots, X_{n}}^{\mathrm{Il}, \mathrm{op}}$ is equivalent to the homotopy colimit $\triangle_{X_{0}, X_{1}}^{\mathrm{op}} \amalg_{\triangle_{X_{1}}^{\mathrm{op}}} \cdots \amalg_{\triangle_{X_{n-1}}^{\mathrm{op}}} \triangle_{X_{n-1}, X_{n}}^{\mathrm{op}}$, i.e. the corresponding iterated pushout in the $\infty$-category $\mathrm{Opd}_{\infty}^{\mathrm{ns}, \mathrm{gen}}$.

Proof. Pullbacks in $\mathrm{Set}_{\Delta}^{+}$preserve colimits, so since $\triangle^{\amalg, \mathrm{op}}$ is a colimit we may identify $\triangle_{X_{0}, \ldots, X_{n}}^{\amalg, o p}$ with the corresponding (ordinary) colimit. But since this colimit can be written as an iterated pushout along cofibrations, it is a homotopy colimit. 
Corollary 6.19. For any spaces $X_{0}, \ldots, X_{n}$, the inclusion

$$
\triangle_{X_{0}, \ldots, X_{n}}^{\mathrm{\amalg}, \mathrm{op}} \hookrightarrow \mathbb{N}_{X_{0}, \ldots, X_{n}}^{\mathrm{op}}
$$

is a trivial cofibration in $\left(\operatorname{Set}_{\Delta}^{+}\right)_{\mathfrak{O}_{\mathrm{ns}}^{\mathrm{gen}}}$.

Proof. The proof of [Hau14b, Proposition 4.22] implies that the inclusion $\triangle_{/[n]}^{\amalg, o p} \hookrightarrow$ $\mathbb{\Lambda}_{/[n]}^{\text {op }}$ is a transfinite composite of pushouts of the morphisms described in Proposition 6.16. Since $\operatorname{Set}_{\Delta}^{+}$is locally Cartesian closed, it follows that for any spaces $X_{0}$, $\ldots, X_{n}$, the inclusion $\triangle_{X_{0}, \ldots, X_{n}}^{\amalg, \mathrm{op}} \hookrightarrow \wedge_{X_{0}, \ldots, X_{n}}^{\mathrm{op}}$ is a transfinite composite of pushouts along pullbacks of such maps. Since the projection $\mathbb{N}_{X_{0}, \ldots, X_{n}}^{\mathrm{op}} \rightarrow \mathbb{N}_{/[n]}^{\mathrm{op}}$ is a fibration in this model structure, it follows from Proposition 6.16 that this map is a trivial cofibration.

Corollary 6.20. Let $\mathcal{V}$ be a monoidal $\infty$-category compatible with small colimits. Then the Segal map

$$
\mathfrak{A} \mathfrak{L G}_{\text {cat }}(\mathcal{V})_{n} \rightarrow \mathfrak{A} \mathfrak{L} \mathfrak{G}_{\text {cat }}(\mathcal{V})_{1} \times_{\mathfrak{A L G}_{\text {cat }}(\mathcal{V})_{0}} \cdots \times_{\mathfrak{A L}_{\text {cat }}(\mathcal{V})_{0}} \mathfrak{A} \mathfrak{L} \mathfrak{G}_{\text {cat }}(\mathcal{V})_{1}
$$

is an equivalence of $\infty$-categories.

Proof. This is a map of Cartesian fibrations over $\mathcal{S}^{\times(n+1)}$, so it suffices to show that for all spaces $X_{0}, \ldots, X_{n}$ the induced map on fibres over $\left(X_{0}, \ldots, X_{n}\right)$ is an equivalence. But this map can be identified with the composite

$$
\begin{aligned}
\left(\mathfrak{A} \mathfrak{L G}_{\text {cat }}\right. & \left.(\mathcal{V})_{n}\right)_{\left(X_{0}, \ldots, X_{n}\right)} \\
& \rightarrow \operatorname{Alg}_{\triangle_{X_{0}, \ldots, X_{n}}^{\mathrm{op}}}(\mathcal{V}) \\
& \rightarrow \operatorname{Alg}_{\triangle_{X_{0}, \ldots, X_{n}}^{\mathrm{I}, \mathrm{op}}}(\mathcal{V}) \\
& \rightarrow \operatorname{Alg}_{\triangle_{X_{0}, X_{1}}^{\mathrm{op}}}(\mathcal{V}) \times_{\operatorname{Alg}_{\triangle_{X_{1}} \mathrm{op}}(\mathcal{V})} \cdots \times_{\operatorname{Alg}_{\triangle_{X_{n-1}}^{\mathrm{op}}}(\mathcal{V})} \operatorname{Alg}_{{\triangle_{X_{n-1}, X_{n}}^{\mathrm{op}}}}(\mathcal{V})
\end{aligned}
$$

where the first map is an equivalence by definition, the second by Corollary 6.19, and the third by Lemma 6.18 .

Combining Corollary 6.20 with Corollary 6.15, we have proved:

Theorem 6.21. Let $\mathcal{V}$ be a monoidal $\infty$-category compatible with small colimits. Then the projection $\mathfrak{A} \mathfrak{L G}_{\text {cat }}(\mathcal{V}) \rightarrow \triangle^{\mathrm{op}}$ is a double $\infty$-category.

Definition 6.22. We say a $\triangle_{X_{0}, \ldots, X_{n}}^{\mathrm{pp}}$-algebra $\mathcal{M}$ in $\mathcal{V}$ is complete if for each $i=$ $0, \ldots, n$ the $\triangle_{X_{i}}^{\text {op }}$-algebra $\sigma_{i}^{*} \mathcal{M}$ is complete, where $\sigma_{i}:[0] \rightarrow[n]$ is the map sending 0 to $i$. We define $\mathfrak{C A T}(\mathcal{V})$ to be the full subcategory of $\mathfrak{A} \mathfrak{L G}_{\text {cat }}(\mathcal{V})$ spanned by the complete composite $\Delta_{X_{0}, \ldots, X_{n}}^{\text {op }}$-algebras for all spaces $X_{0}, \ldots, X_{n}$.

Corollary 6.23. The projection $\mathfrak{C A T}(\mathcal{V}) \rightarrow \mathbb{\Delta}^{\mathrm{op}}$ is a double $\infty$-category.

Proof. To see that $\mathfrak{C A T}(\mathcal{V}) \rightarrow \mathbb{\Delta}^{\text {op }}$ is coCartesian, it suffices to observe that given a complete $\triangle_{X_{0}, \ldots, X_{n}}^{\text {op }}$-algebra $M: \triangle_{X_{0}, \ldots, X_{n}}^{\mathrm{op}} \rightarrow \mathcal{V}^{\otimes}$ and a morphism $\phi:[m] \rightarrow[n]$ in $\triangle$, the target $\phi^{*} M: \triangle_{X_{\phi(0)}, \ldots, X_{\phi(m)}}^{\mathrm{op}} \rightarrow \mathcal{V}^{\otimes}$ of the coCartesian morphism over $\phi$ with source $M$ is also complete, since $\sigma_{i}^{*} \phi^{*} M \simeq \sigma_{\phi(i)}^{*} M$ and this is complete. 
To see that it is moreover a double $\infty$-category, observe that

$$
\mathfrak{C A T}(\mathcal{V})_{1} \simeq \operatorname{Cat}_{\infty}^{\mathcal{V}} \times_{\mathfrak{A L G} \dot{G}_{\text {cat }}}(\mathcal{V})_{0} \mathfrak{A} \mathfrak{L} \mathfrak{G}_{\text {cat }}(\mathcal{V})_{1} \times_{\mathfrak{A} \mathfrak{L} \mathfrak{G}_{\text {cat }}}(\mathcal{V})_{0} \operatorname{Cat}_{\infty}^{\mathcal{V}}
$$

and so under the identification of Corollary 6.20 , the full subcategory $\mathfrak{C A T}(\mathcal{V})_{n}$ of $\mathfrak{A} \mathfrak{G}_{\text {cat }}(\mathcal{V})_{n}$ precisely corresponds to the iterated fibre product

$$
\mathfrak{C A T}(\mathcal{V})_{1} \times_{\mathfrak{C A T}(\mathcal{V})_{0}} \cdots \times_{\mathfrak{C A T}(\mathcal{V})_{0}} \mathfrak{C A T}(\mathcal{V})_{1}
$$

\section{Natural transformations}

In this section we consider the obvious definition of natural transformations between functors of enriched $\infty$-categories. We then use this to construct $\infty$-categories of functors and show that these are the underlying $\infty$-categories of the internal Hom when this exists.

Definition 7.1. We may regard the categories $[n]$ as (levelwise discrete) Segal spaces, and thus as $\mathcal{S}$-enriched $\infty$-categories via the equivalence of [GH15, Theorem 4.4.7]. If $\mathcal{V}$ is a monoidal $\infty$-category compatible with small colimits, then $\operatorname{Alg}_{\text {cat }}(\mathcal{V})$ is tensored over $\operatorname{Alg}_{\text {cat }}(\mathcal{S})$ by [GH15, Corollary 4.3.17], so for any $\mathcal{V}$ - $\infty$-category $\mathcal{C}$ we have $\mathcal{V}$ - $\infty$-categories $\mathcal{C} \otimes[n] .{ }^{5}$ If $f, g: \mathcal{C} \rightarrow \mathcal{D}$ are functors of $\mathcal{V}$ - $\infty$-categories, a natural transformation from $f$ to $g$ is a functor $\eta: \mathcal{C} \otimes[1] \rightarrow \mathcal{D}$ such that $\eta \circ\left(\operatorname{ide} \otimes d_{1}\right) \simeq f$ and $\eta \circ\left(\operatorname{id}_{\mathcal{C}} \otimes d_{0}\right) \simeq g$.

Given this definition of natural transformations, there is an obvious simplicial space that should be the $\infty$-category of $\mathcal{V}$-functors between two $\mathcal{V}$ - $\infty$-categories:

Definition 7.2. Suppose $\mathcal{C}$ and $\mathcal{D}$ are $\mathcal{V}$ - $\infty$-categories. We let $\operatorname{Fun}_{\mathcal{V}}(\mathcal{C}, \mathcal{D})$ denote the simplicial space $\mathbb{\triangle}^{\mathrm{op}} \rightarrow \mathcal{S}$ sending $[n]$ to $\operatorname{Map}_{\mathrm{Cat}_{\infty}^{\mathrm{v}}}(\mathcal{C} \otimes[n], \mathcal{D})$.

Our first goal in this section is to check that this is indeed a Segal space, and that it's complete if the target is a complete $\mathcal{V}$ - $\infty$-category:

Proposition 7.3. Let $\mathcal{V}$ be a monoidal $\infty$-category compatible with small colimits, and let $\mathcal{C}$ and $\mathcal{D}$ be $\mathcal{V}$ - $\infty$-categories.

(i) The simplicial space $\operatorname{Fun}_{\mathcal{V}}(\mathcal{C}, \mathcal{D})$ is a Segal space.

(ii) For any Segal space $X$ we have a natural equivalence

$$
\operatorname{Map}_{\operatorname{Seg}_{\infty}}\left(X, \operatorname{Fun}_{\mathcal{V}}(\mathcal{C}, \mathcal{D})\right) \simeq \operatorname{Map}_{\operatorname{Alg}_{\text {cat }}(\mathcal{V})}(\mathcal{C} \otimes X, \mathcal{D}),
$$

where on the right we regard $X$ as an $\mathcal{S}-\infty$-category.

(iii) The underlying groupoid object $\iota \operatorname{Fun}_{\mathcal{V}}(\mathcal{C}, \mathcal{D})$ of the Segal space $\operatorname{Fun}_{\mathcal{V}}(\mathcal{C}, \mathcal{D})$ is $\operatorname{Map}\left(\mathcal{C} \otimes E^{\bullet}, \mathcal{D}\right)$. (Thus the "correct" space of objects of $\operatorname{Fun}_{\mathcal{V}}(\mathcal{C}, \mathcal{D})$ is the geometric realization $\left.\left|\operatorname{Map}\left(\mathcal{C} \otimes E^{\bullet}, \mathcal{D}\right)\right|.\right)$

(iv) If $\mathcal{D}$ is a complete $\mathcal{V}-\infty$-category, then the Segal space $\operatorname{Fun}_{\mathcal{V}}(\mathcal{C}, \mathcal{D})$ is complete.

${ }^{5}$ In fact, we may define $\mathcal{C} \otimes[n]$ as a $\mathcal{V}$ - $\infty$-category provided only that $\mathcal{V}$ has an initial object and this is compatible with the tensor product, but we will not need this generality. 
Proof. Tensoring $\mathcal{V}$ - $\infty$-categories with $\mathcal{S}$ - $\infty$-categories preserves colimits in each variable by [GH15, Corollary 4.3.17], so to prove (i) it suffices to show that the $\mathcal{S}$ - $\infty$-categories $[n]$ form a coSegal object, i.e. that the natural maps $[1] \amalg_{[0]} \cdots \amalg_{[0]}[1] \rightarrow[n]$ are equivalences in $\operatorname{Alg}_{\text {cat }}(\mathcal{S})$.

Recall from $[\mathrm{GH15}, \S 3.3]$ that there is a free-forgetful adjunction between $\mathcal{S}$ - $\infty$ categories and $\mathcal{S}$-graphs, where an $\mathcal{S}$-graph with space of objects $X$ is just a functor $X \times X \rightarrow \mathcal{S}$. Let $\mathcal{G}_{n}$ denote the $\mathcal{S}$-graph with objects $\{0, \ldots, n\}$ and

$$
\mathcal{S}_{n}(i, j)= \begin{cases}*, & i<j \\ \emptyset, & j \geqslant i .\end{cases}
$$

Then it is easy to see that $[n]$ is the free $\mathcal{S}$ - $\infty$-category on the graph $\mathcal{G}_{n}$. Moreover, it is obvious that the map $\mathcal{G}_{1} \amalg_{\mathcal{G}_{0}} \cdots \amalg_{\mathcal{G}_{0}} \mathcal{G}_{1} \rightarrow \mathcal{G}_{n}$ is an equivalence of $\mathcal{S}$-graphs. Since the

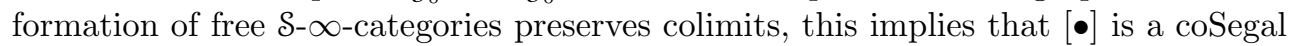
object, which proves (i).

Every Segal space can be canonically written as a colimit of a diagram of the objects $[n]$. Specifically, the Segal space $X$ is the coend of

$$
\bar{X}: \triangle \times \mathbb{\Delta}^{\mathrm{op}} \rightarrow \operatorname{Seg}_{\infty}, \quad([n],[m]) \mapsto \operatorname{colim}_{X_{m}}[n] .
$$

Since $\operatorname{Map}\left([n], \operatorname{Fun}_{\mathcal{V}}(\mathcal{C}, \mathcal{D})\right) \simeq \operatorname{Map}(\mathcal{C} \otimes[n], \mathcal{D})$ we then have

$$
\begin{aligned}
\operatorname{Map}\left(X, \operatorname{Fun}_{\mathcal{V}}(\mathcal{C}, \mathcal{D})\right) & \simeq \operatorname{Map}\left(\operatorname{colim}_{\operatorname{Tw}(\triangle)} \bar{X}, \operatorname{Fun}_{\mathcal{V}}(\mathcal{C}, \mathcal{D})\right) \\
& \simeq \lim _{\operatorname{Tw}(\triangle)} \operatorname{Map}\left(\bar{X}, \operatorname{Fun}_{\mathcal{V}}(\mathcal{C}, \mathcal{D})\right) \\
& \simeq \lim _{\operatorname{Tw}(\triangle)} \operatorname{Map}(\mathcal{C} \otimes \bar{X}, \mathcal{D}) \\
& \simeq \operatorname{Map}(\mathcal{C} \otimes X, \mathcal{D}),
\end{aligned}
$$

which proves (ii).

The underlying groupoid object of a Segal space $X$ is $\operatorname{Map}\left(E^{\bullet}, X\right)$. By (ii), the underlying groupoid object of $\operatorname{Fun}_{\mathcal{V}}(\mathcal{C}, \mathcal{D})$ is therefore $\operatorname{Map}\left(\mathcal{C} \otimes E^{\bullet}, \mathcal{D}\right)$, which proves (iii). The Segal space $\operatorname{Fun}_{\mathcal{V}}(\mathcal{C}, \mathcal{D})$ is complete if and only if the canonical map from the colimit of this simplicial space to $\operatorname{Fun}_{\mathcal{V}}(\mathcal{C}, \mathcal{D})_{0}$ is an equivalence. By [GH15, Corollary 5.5.10] we know that if $\mathcal{D}$ is complete then

$$
\left|\iota \operatorname{Fun}_{\mathcal{V}}(\mathcal{C}, \mathcal{D})\right| \simeq \operatorname{Map}(\mathcal{C}, \mathcal{D}) \simeq \operatorname{Fun}_{\mathcal{V}}(\mathcal{C}, \mathcal{D})_{0},
$$

thus $\operatorname{Fun}_{\mathcal{V}}(\mathcal{C}, \mathcal{D})$ is complete.

Now suppose $\mathcal{V}$ is a presentably symmetric monoidal $\infty$-category, i.e. a symmetric monoidal $\infty$-category compatible with small colimits such that the underlying $\infty$-category is presentable. Then by [GH15, Corollary 4.3.16] and [GH15, Proposition 5.7.16] the $\infty$-categories $\operatorname{Alg}_{\text {cat }}(\mathcal{V})$ and $\mathrm{Cat}_{\infty}^{\mathcal{V}}$ are also presentably symmetric monoidal. This implies that $\operatorname{Alg}_{\text {cat }}(\mathcal{V})$ and $\mathrm{Cat}_{\infty}^{\mathcal{V}}$ have internal Hom objects; we write $\mathcal{D}^{\mathcal{C}}$ for the internal Hom for maps $\mathcal{C} \rightarrow \mathcal{D}$ in $\operatorname{Alg}_{\text {cat }}(\mathcal{V})$.

Let's check that the underlying $\infty$-category of the internal Hom $\mathcal{D}^{\mathbb{C}}$ is precisely the functor $\infty$-category $\operatorname{Fun}_{\mathcal{V}}(\mathcal{C}, \mathcal{D})$ :

Proposition 7.4. Let $\mathcal{V}$ be a presentably symmetric monoidal $\infty$-category, and suppose $\mathcal{C}$ and $\mathcal{D}$ are $\mathcal{V}$ - $\infty$-categories. 
(i) If $\mathcal{D}$ is complete, then the $\mathcal{V}$ - $\infty$-category $\mathcal{D}^{\mathcal{C}}$ is complete for any $\mathcal{C}$. Moreover, $\mathcal{D}^{\mathbb{C}}$ is also the internal Hom in $\mathrm{Cat}_{\infty}^{\mathcal{D}}$.

(ii) Write $t: \mathcal{S} \rightarrow \mathcal{V}$ for the unique colimit-preserving strong monoidal functor sending * to the unit I; by [GH15, Proposition A.81] this has a lax monoidal right adjoint $u: \mathcal{V} \rightarrow \mathcal{S}$ given by $\operatorname{Map}(I,-)$. Then $\operatorname{Map}\left(E^{0} \otimes[\bullet], \mathcal{C}\right)$ is the Segal space corresponding to the $\mathcal{S}$ - $\infty$-category $u_{*} \mathrm{C}$.

(iii) The Segal space corresponding to the $\mathcal{S}-\infty$-category $u_{*} \mathcal{D}^{\mathcal{C}}$ underlying the internal Hom is $\operatorname{Fun}^{\mathcal{V}}(\mathcal{C}, \mathcal{D})$.

Proof. To prove (i), we must show that $\operatorname{Map}\left(E^{0}, \mathcal{D}^{\mathfrak{C}}\right) \rightarrow \operatorname{Map}\left(E^{1}, \mathcal{D}^{\mathbb{C}}\right)$ is an equivalence. Passing to left adjoints this is $\operatorname{Map}(\mathcal{C}, \mathcal{D}) \rightarrow \operatorname{Map}\left(E^{1} \otimes \mathcal{C}, \mathcal{D}\right)$, which is an equivalence since $\mathcal{C} \otimes E^{1} \rightarrow \mathcal{C}$ is a local equivalence by [GH15, Proposition 4.45].

Since $\mathcal{D}^{\mathcal{C}}$ is complete we have, for any complete $\mathcal{V}$ - $\infty$-category $\mathcal{A}$,

$$
\begin{aligned}
\operatorname{Map}_{\text {Cat }}\left(\mathcal{A}, \mathcal{D}^{\mathcal{C}}\right) & \simeq \operatorname{Map}_{\operatorname{Alg}_{\text {cat }}(\mathcal{V})}\left(\mathcal{A}, \mathcal{D}^{\mathcal{C}}\right) \simeq \operatorname{Map}_{\operatorname{Alg}_{\text {cat }}(\mathcal{V})}(\mathcal{A} \otimes \mathcal{C}, \mathcal{D}) \\
& \simeq \operatorname{Map}_{\mathrm{Cat}}{ }_{\infty}^{\mathcal{D}}(\mathcal{A} \otimes \mathcal{C}, \mathcal{D}),
\end{aligned}
$$

hence $\mathcal{D}^{\mathcal{C}}$ is also the internal hom in $\mathrm{Cat}_{\infty}^{\mathcal{D}}$.

To prove (ii), observe that the Segal space corresponding to $u_{*} \mathcal{C}$ is

$$
\operatorname{Map}_{\operatorname{Alg}_{\text {cat }}(\mathcal{S})}\left([n], u_{*} \mathcal{C}\right) \simeq \operatorname{Map}_{\mathrm{Alg}_{\text {cat }}(\mathcal{V})}\left(t_{*}[n], \mathcal{C}\right) \simeq \operatorname{Map}_{\operatorname{Alg}_{\text {cat }}(\mathcal{V})}\left(E^{0} \otimes[n], \mathcal{C}\right) .
$$

Thus the Segal space associated to $u_{*} \mathcal{C}^{\mathcal{D}}$ is given by

$$
\operatorname{Map}_{\operatorname{Alg}_{\text {cat }}(\mathcal{V})}\left(E^{0} \otimes[\bullet], \mathcal{D}^{\mathcal{C}}\right) \simeq \operatorname{Map}_{\operatorname{Alg}_{\text {cat }}(\mathcal{V})}(\mathcal{C} \otimes[\bullet], \mathcal{D}) \simeq \operatorname{Fun}_{\mathcal{V}}(\mathcal{C}, \mathcal{D})
$$

\section{The $(\infty, 2)$-category of enriched $\infty$-categories}

The double $\infty$-category $\mathfrak{A} \mathfrak{L G}_{\text {cat }}(\mathcal{V})$ has two underlying $(\infty, 2)$-categories: one where the 1-morphisms are bimodules, and one where they are functors; we write $\mathrm{ALG}_{\mathrm{cat}}(\mathcal{V})$ for the latter. Our goal in this section is to show that the 2-morphisms in $\mathrm{ALG}_{\mathrm{cat}}(\mathcal{V})$ can be identified with natural transformations, as we defined them in the previous section. More precisely, we'll show:

Proposition 8.1. Let $\mathcal{C}$ and $\mathcal{D}$ be $\mathcal{V}-\infty$-categories. There is a natural equivalence

$$
\operatorname{ALG}_{\text {cat }}(\mathcal{V})(\mathcal{C}, \mathcal{D}) \simeq \operatorname{Fun}_{\mathcal{V}}(\mathcal{C}, \mathcal{D}) .
$$

Remark 8.2. The two underlying $(\infty, 2)$-categories of a double $\infty$-category $X \rightarrow \triangle^{\text {op }}$ can be described as follows: If $F: \triangle^{\mathrm{op}} \rightarrow$ Cat $_{\infty}$ is the associated functor, then regarding Cat $_{\infty}$ as the $\infty$-category of complete Segal spaces allows us to think of $F$ as a double Segal space $\Delta^{\mathrm{op}} \times \Delta^{\mathrm{op}} \rightarrow \mathcal{S}$. Now we can take the underlying 2 -fold Segal space in either direction, which amounts to replacing $F_{n m}$ by its pullback along the degeneracy map from $F_{00}$ to $F_{n 0}$ or $F_{m 0}$ - see [Hau14a, Proposition 2.13] for a precise statement.

If $X$ is a space, let's abbreviate $\triangle_{X}^{\mathrm{op}}[n]:=\triangle_{X, \ldots, X}^{\mathrm{op}} \rightarrow \triangle_{/[n]}^{\mathrm{op}}$. Let $\pi_{n}:[n] \rightarrow[0]$ be the unique map; this induces maps $\pi_{n, *}: \triangle_{X}^{\mathrm{op}}[n] \rightarrow \triangle_{X}^{\mathrm{op}}$ and thus $\pi_{n}^{*}: \mathfrak{A} \mathfrak{L} \mathfrak{G}_{\text {cat }}(\mathcal{V})_{0} \rightarrow$ $\mathfrak{A} \mathfrak{L} \mathfrak{G}_{\text {cat }}(\mathcal{V})_{n}$. If $\mathcal{C}$ and $\mathcal{D}$ are $\mathcal{V}$ - $\infty$-categories, the $\infty$-category of maps from $\mathcal{C}$ to $\mathcal{D}$ in 
$\operatorname{ALG}_{\text {cat }}(\mathcal{V})$ can be identified with the Segal space

$$
[n] \mapsto \operatorname{Map}_{\mathfrak{A} \mathfrak{L} \mathfrak{G}_{\text {cat }}(\mathcal{V})_{n}}\left(\pi_{n}^{*} \mathcal{C}, \pi_{n}^{*} \mathcal{D}\right) .
$$

Our goal is then to show that there is a natural equivalence between the spaces $\operatorname{Map}_{\mathfrak{A} \mathfrak{L} \mathfrak{G}_{\text {cat }}(\mathcal{V})_{n}}\left(\pi_{n}^{*} \mathcal{C}, \pi_{n}^{*} \mathcal{D}\right)$ and $\operatorname{Map}_{\mathrm{Alg}_{\text {cat }}(\mathcal{V})}(\mathcal{C} \otimes[n], \mathcal{D})$. To do this we'll relate the $\triangle_{X}^{\mathrm{op}}[n]$-algebra $\pi_{n}^{*} \mathcal{C}$ to the $\triangle_{X \times\{0, \ldots, n\}}^{\mathrm{op}}$-algebra $\mathcal{C} \otimes[n]$.

Let $I_{n}: \triangle_{/[n]}^{\mathrm{op}} \rightarrow \triangle_{\{0, \ldots, n\}}^{\mathrm{op}}$ denote the obvious inclusion, given on objects by sending $\phi:[m] \rightarrow[n]$ to the list $(\phi(0), \ldots, \phi(m))$, and write $\xi_{n}$ for the projection $\triangle_{\{0, \ldots, n\}}^{\mathrm{op}} \rightarrow$ $\triangle^{\mathrm{op}}$. Then for any space $X$ we have a commutative diagram

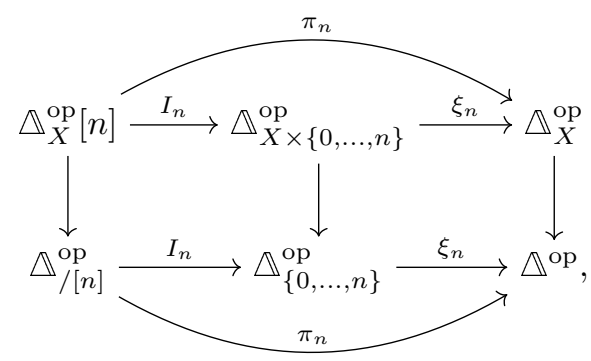

where both squares are Cartesian, and the maps are all morphisms of generalized non-symmetric $\infty$-operads.

Lemma 8.3. For any space $X$, the inclusion $I_{n}: \triangle_{X}^{\mathrm{op}}[n] \rightarrow \triangle_{X \times\{0, \ldots, n\}}^{\mathrm{op}}$ is extendable.

Proof. For $\xi \in \triangle_{X \times\{0, \ldots, n\}}^{\mathrm{op}}$, the $\infty$-category $\triangle_{X}^{\mathrm{op}}[n]_{/ \xi}^{\text {act }}$ is empty if $\xi \notin \triangle_{X}^{\mathrm{op}}[n]$, or has a final object if $\xi \in \triangle_{X}^{\mathrm{op}}[n]$. The extendability condition follows immediately from this.

Suppose $\mathcal{V}$ is a monoidal $\infty$-category compatible with small colimits, and let $\mathcal{C}$ be a $\mathcal{V}$ - $\infty$-category with space of objects $X$. Tensoring $\mathcal{C}$ with the map $[n] \rightarrow[0]$ we get a map $\mathcal{C} \otimes[n] \rightarrow \mathcal{C}$ lying over the projection $X \times\{0, \ldots, n\} \rightarrow X$. Thus this gives a map $\mathcal{C} \otimes[n] \rightarrow \xi_{n}^{*} \mathcal{C}$ of $\triangle_{X \times\{0, \ldots, n\}}^{\mathrm{op}}$-algebras.

Lemma 8.4. In the situation above, the induced map

$$
I_{n}^{*}(\mathcal{C} \otimes[n]) \rightarrow I_{n}^{*} \xi_{n}^{*} \simeq \pi_{n}^{*} \mathcal{C}
$$

is an equivalence.

Proof. It suffices to observe that for $i \leqslant j$ and any $x, y \in X$ the morphism

$$
(\mathcal{C} \otimes[n])((x, i),(y, j)) \rightarrow \mathcal{C}(x, y)
$$

is an equivalence.

This map therefore has an inverse $\pi_{n}^{*} \mathcal{C} \rightarrow I_{n}^{*}(\mathcal{C} \otimes[n])$, and since $I_{n}^{*}$ has a left adjoint by Lemma 8.3 there is a natural map $I_{n, !} \pi_{n}^{*} \mathcal{C} \rightarrow \mathcal{C} \otimes[n]$ of $\triangle_{X \times\{0, \ldots, n\}}^{\text {op }}$-algebras.

Proposition 8.5. In the situation above, the morphism $I_{n, !} \pi_{n}^{*} \mathrm{C} \rightarrow \mathcal{C} \otimes[n]$ is an equivalence.

Proof. Again observe that for $\xi \in \triangle_{X \times\{0, \ldots, n\}}^{\mathrm{op}}$, the $\infty$-category $\triangle_{X}^{\mathrm{op}}[n]_{/ \xi}^{\text {act }}$ is empty if $\xi \notin \mathbb{\triangle}_{X}^{\mathrm{op}}[n]$, or has a final object if $\xi \in \mathbb{\triangle}_{X}^{\mathrm{op}}[n]$. By the definition of left operadic Kan 
extensions we therefore see that for $x, y \in X$ and $i, j \in\{0, \ldots, n\}$ we have

$$
I_{n, !} \pi_{n}^{*} \mathcal{C}((x, i),(y, j)) \simeq \begin{cases}\emptyset, & i>j \\ \mathcal{C}(x, y), & i \leqslant j .\end{cases}
$$

The forgetful functor from $\triangle_{X \times\{0, \ldots, n\}}^{\text {op }}$-algebras to functors $(X \times\{0, \ldots, n\})^{\times 2} \rightarrow \mathcal{V}$ is conservative by [GH15, Lemma A.5.5], so this completes the proof.

Now consider the algebra fibration $\operatorname{Alg}(\mathcal{V}) \rightarrow \operatorname{Opd}_{\infty}^{\text {ns,gen }}$. Since $\mathfrak{A} \mathfrak{L} \mathfrak{G}_{\text {cat }}(\mathcal{V})_{n}$ is pulled back from this, if $\mathcal{C}$ and $\mathcal{D}$ are $\mathcal{V}$ - $\infty$-categories with spaces of objects $X$ and $Y$, respectively, then we have a pullback square

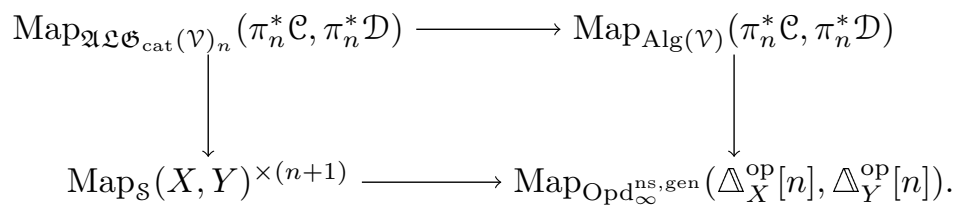

By Proposition 8.5 we also have a natural equivalence

$$
\operatorname{Map}_{\operatorname{Alg}(\mathcal{V})}\left(\pi_{n}^{*} \mathcal{C}, \pi_{n}^{*} \mathcal{D}\right) \simeq \operatorname{Map}_{\operatorname{Alg}(\mathcal{V})}\left(I_{n, !} \pi_{n}^{*} \mathcal{C}, \xi_{n}^{*} \mathcal{D}\right) .
$$

Moreover, if we consider the diagram

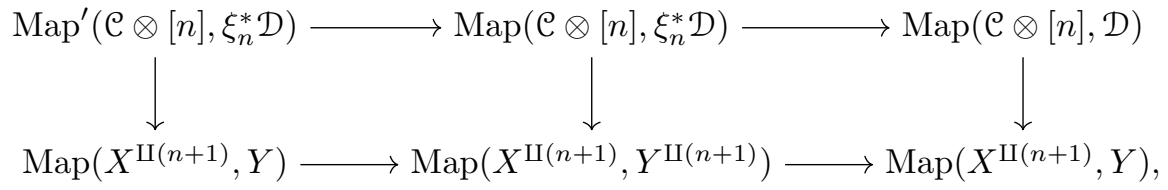

where the left square is defined to be a pullback square, then the top composite map is an equivalence: since the bottom composite map is the identity, it suffices to check that the map is an equivalence on each fibre, which is clear. Thus we have identified both $\operatorname{Map}_{\operatorname{Alg}(\mathcal{V})}\left(\pi_{n}^{*} \mathcal{C}, \pi_{n}^{*} \mathcal{D}\right)$ and $\operatorname{Map}(\mathcal{C} \otimes[n], \mathcal{D})$ with the same pullback, which completes the proof of Proposition 8.1.

Corollary 8.6. Let $\mathrm{CAT}_{\infty}^{\mathcal{V}}$ be the underlying 2-fold Segal space of $\mathfrak{C A T}(\mathcal{V})$ with functors as 1-morphisms. Then this 2-fold Segal space is complete.

Proof. The underlying Segal space of $\mathrm{CAT}_{\infty}^{\mathcal{V}}$ is that associated to the $\infty$-category $\mathrm{Cat}_{\infty}^{\mathcal{D}}$, and so is complete. Using the completeness criterion of [Hau14a, Theorem 5.18] it then suffices to show that the Segal space $\operatorname{CAT}_{\infty}^{\mathcal{V}}(\mathcal{C}, \mathcal{D})$ of maps from $\mathcal{C}$ to $\mathcal{D}$ is complete for all complete $\mathcal{V}$ - $\infty$-categories $\mathcal{C}$ and $\mathcal{D}$, which follows from combining Proposition 8.1 and Proposition 7.3.

\section{Functoriality and monoidal structures}

In this section we consider the functoriality in $\mathcal{V}$ of the double $\infty$-category of $\mathcal{V}$ $\infty$-categories. Here we restrict ourselves to the "algebraic" or pre-localized case of the double $\infty$-categories $\mathfrak{A} \mathfrak{L} \mathfrak{G}_{\text {cat }}(\mathcal{V})$ - since composition with a colimit-preserving monoidal functor does not usually preserve complete objects (cf. [GH15, §5.7]), to 
establish functoriality for the double $\infty$-categories $\mathfrak{C A T}(\mathcal{V})$ we must first show that the $\infty$-category of $\mathcal{C}$ - $\mathcal{D}$-bimodules in $\mathcal{V}$ is invariant under fully faithful and essentially surjective functors of $\mathcal{C}$ and $\mathcal{D}$. This result is most naturally proved as a consequence of the Yoneda Lemma (in the form of the representability of the $\infty$-category of bimodules), and so we postpone it to a sequel to this paper.

Definition 9.1. In $\S 6$ we constructed a functor $\Delta_{\mathcal{S}} \rightarrow \operatorname{Opd}_{\infty}^{\text {ns,gen }}$ that sends $\left(X_{0}, \ldots, X_{n}\right)$ to $\triangle_{X_{0}, \ldots, X_{n}}^{\mathrm{op}}$. Combining this with the algebra functor

$$
\mathrm{Alg}:\left(\mathrm{Opd}_{\infty}^{\mathrm{ns}, \mathrm{gen}}\right)^{\mathrm{op}} \times \mathrm{Opd}_{\infty}^{\mathrm{ns}, \mathrm{gen}} \rightarrow \mathrm{Cat}_{\infty},
$$

we get a functor $\left(\triangle_{S}\right)^{\text {op }} \times \operatorname{Opd}_{\infty}^{\text {ns,gen }} \rightarrow$ Cat $_{\infty}$ that sends the object $\left(\left(X_{0}, \ldots, X_{n}\right), \mathcal{O}\right)$ to $\operatorname{Alg}_{\triangle_{X_{0}, \ldots, X_{n}}^{o p}}(\mathcal{O})$. Let $\overline{\mathfrak{A} \mathfrak{L} \mathfrak{G}_{\text {cat }}} v^{\infty} \rightarrow \triangle_{\mathcal{S}} \times\left(\mathrm{Opd}_{\infty}^{\text {ns,gen }}\right)^{\text {op }}$ be a Cartesian fibration associated to this functor, and then take $\overline{\mathfrak{A L G}}_{\text {cat }} \rightarrow \triangle^{\mathrm{op}} \times \mathrm{Opd}_{\infty}^{\mathrm{ns}, \mathrm{gen}}$ to be a coCartesian fibration associated to the composite

$$
\overline{\mathfrak{A L G}}_{\text {cat }}^{\vee} \rightarrow \Delta_{\mathcal{S}} \times\left(\mathrm{Opd}_{\infty}^{\mathrm{ns}, \mathrm{gen}}\right)^{\mathrm{op}} \rightarrow \triangle \times\left(\mathrm{Opd}_{\infty}^{\mathrm{ns}, \mathrm{gen}}\right)^{\mathrm{op}} .
$$

Remark 9.2. The coCartesian fibration $\overline{\mathfrak{A L G}}_{\text {cat }} \rightarrow \triangle^{\mathrm{op}} \times \mathrm{Opd}_{\infty}^{\text {ns,gen }}$ determines a functor $\Delta^{\mathrm{op}} \times \mathrm{Opd}_{\infty}^{\mathrm{ns}, \text { gen }} \rightarrow \mathrm{Cat}_{\infty}$ or $\mathrm{Opd}_{\infty}^{\mathrm{ns}, \mathrm{gen}} \rightarrow \mathrm{Fun}\left(\Delta^{\mathrm{op}}, \mathrm{Cat}_{\infty}\right)$.

Definition 9.3. Let $\widehat{\mathrm{Cat}}_{\infty}^{\mathrm{coC}}$ denote the $\infty$-category of (large) $\infty$-categories with small colimits and colimit-preserving functors. This $\infty$-category has a tensor product, constructed in $[\operatorname{Lur} 14, \S 4.8 .1]$, such that a map $\mathcal{C} \otimes \mathcal{D} \rightarrow \mathcal{E}$ is equivalent to a map $\mathcal{C} \times \mathcal{D} \rightarrow \mathcal{E}$ that preserves colimits separately in each variable. Then the $\infty$-category $\operatorname{Mon}_{\infty}^{\mathrm{coC}}:=\operatorname{Alg}_{\mathbb{E}_{1}}^{\Sigma}\left(\widehat{\mathrm{Cat}}_{\infty}^{\mathrm{coC}}\right)$ of associative algebras with respect to this tensor product is the $\infty$-category of monoidal $\infty$-categories compatible with small colimits and colimit-preserving monoidal functors.

Definition 9.4. There is a forgetful functor $\operatorname{Mon}_{\infty}^{\mathrm{coC}} \rightarrow \widehat{\mathrm{Opd}}_{\infty}^{\mathrm{ns}, \text { gen }}$. Let $\overline{\mathfrak{A L G}}_{\text {cat }}^{\prime} \rightarrow$ $\Delta^{\mathrm{op}} \times \operatorname{Mon}_{\infty}^{\mathrm{coC}}$ be defined by the pullback along this of the obvious variant of the

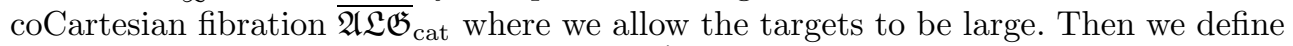
$\mathfrak{A} \mathfrak{L G}_{\text {cat }}$ to be the full subcategory of $\overline{\mathfrak{A L G}}_{\text {cat }}^{\prime}$ spanned by the objects of $\mathfrak{A} \mathfrak{L} \mathfrak{G}_{\text {cat }}(\mathcal{V})$ for all $\mathcal{V}$ in $\mathrm{Mon}_{\infty}^{\mathrm{coC}}$.

Proposition 9.5. The restricted projection $\mathfrak{A L G}_{\text {cat }} \rightarrow \triangle^{\mathrm{op}} \times \mathrm{Mon}_{\infty}^{\mathrm{coC}}$ is a coCartesian fibration.

Proof. It suffices to prove that if $f: \mathcal{V}^{\otimes} \rightarrow \mathcal{W}^{\otimes}$ is a colimit-preserving monoidal functor then for every composite algebra $M: \triangle_{X_{0}, \ldots, X_{n}}^{\mathrm{op}} \rightarrow \mathcal{V}^{\otimes}$, the composite map $f_{*} M: \triangle_{X_{0}, \ldots, X_{n}}^{\mathrm{op}} \rightarrow \mathcal{V}^{\otimes} \rightarrow \mathcal{W}^{\otimes}$ is also a composite algebra. In other words, we must show that the diagram

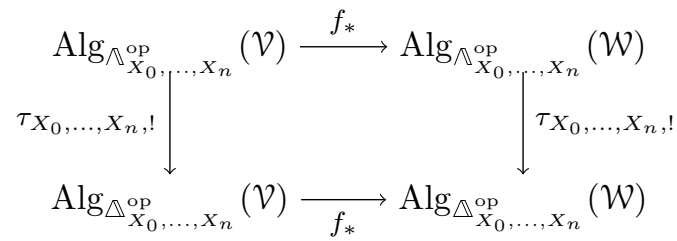

commutes. This is a special case of [GH15, Lemma A.4.7]. 
The coCartesian fibration $\mathfrak{A} \mathfrak{L G}_{\text {cat }} \rightarrow \mathbb{\Delta}^{\mathrm{op}} \times \operatorname{Mon}_{\infty}^{\mathrm{coC}}$ determines a functor $\mathrm{Mon}_{\infty}^{\mathrm{coC}} \times$ $\triangle^{\mathrm{op}} \rightarrow \mathrm{Cat}_{\infty}$, or equivalently $\operatorname{Mon}_{\infty}^{\mathrm{coC}} \rightarrow \operatorname{Fun}\left(\Delta^{\mathrm{op}}, \mathrm{Cat}_{\infty}\right)$. This functor factors through the full subcategory $\mathrm{Cat}\left(\mathrm{Cat}_{\infty}\right)$ spanned by the double $\infty$-categories, giving:

Corollary 9.6. The double $\infty$-category $\mathfrak{A} \mathfrak{L} \mathfrak{G}_{\text {cat }}(\mathcal{V})$ is functorial in colimit-preserving monoidal functors between monoidal $\infty$-categories $\mathcal{V}$ compatible with small colimits, i.e. it is given by a functor $\mathrm{Mon}_{\infty}^{\mathrm{coC}} \rightarrow \operatorname{Cat}\left(\mathrm{Cat}_{\infty}\right)$.

Next we want to show that the functors $\overline{\mathfrak{A L E}}$ cat $(-)$ and $\mathfrak{A L G}_{\text {cat }}(-)$ are lax monoidal. This requires a somewhat more involved construction, as we want the lax monoidal structure to be defined in terms of the "external product" $M \otimes N$ of $M: \triangle_{X_{0}, \ldots, X_{n}}^{\mathrm{op}} \rightarrow$ $\mathcal{V}^{\otimes}$ and $N: \triangle_{Y_{0}, \ldots, Y_{n}}^{\mathrm{op}} \rightarrow \mathcal{W}^{\otimes}$ given by

$$
\begin{aligned}
M \otimes N: \triangle_{X_{0} \times Y_{0}, \ldots, X_{n} \times Y_{n}}^{\mathrm{op}} & \simeq \triangle_{X_{0}, \ldots, X_{n}}^{\mathrm{op}} \times_{\Delta_{/[n]}^{\mathrm{op}}} \triangle_{Y_{0}, \ldots, Y_{n}}^{\mathrm{op}} \\
& \rightarrow \triangle_{X_{0}, \ldots, X_{n}}^{\mathrm{op}} \times_{\Delta^{\mathrm{op}}} \triangle_{Y_{0}, \ldots, Y_{n}}^{\mathrm{op}} \\
& \rightarrow \mathcal{V}^{\otimes} \times_{\Delta^{\mathrm{op}}} \mathcal{W}^{\otimes},
\end{aligned}
$$

which means that we must consider for each $n$ the fibre product of generalized nonsymmetric $\infty$-operads over $\triangle_{/[n]}^{\mathrm{op}}$ in a compatible manner.

Lemma 9.7. Suppose $\mathcal{C}$ is an $\infty$-category with finite colimits. Then the functor $\mathcal{C} \rightarrow$ Cat $_{\infty}$ sending $x$ to $\mathfrak{C}_{x /}$ lifts to a functor from $\mathfrak{C}$ to symmetric monoidal $\infty$-categories sending $x$ to $\left(\mathrm{C}_{x /}\right)^{\amalg_{x}}$. Let $\left(\mathcal{C}_{\bullet} /\right)^{\amalg} \rightarrow \mathcal{C} \times \square^{\mathrm{op}}$ be the coCartesian fibration of $\infty$-operads induced by this; then the forgetful functors $\mathfrak{C}_{x /} \rightarrow \mathcal{C}$ give rise to a morphism of $\infty$ operads $\left(\mathrm{C}_{\bullet} /\right)^{\amalg} \rightarrow \mathrm{e}^{\amalg}$.

Proof. Immediate from [Lur14, Corollary 2.4.3.11 and Proposition 2.4.3.16].

Construction 9.8. We can apply Lemma 9.7 to $\left(\mathrm{Opd}_{\infty}^{\mathrm{ns}, \mathrm{gen}}\right)^{\text {op }}$ to get a morphism of $\infty$-operads $\left(\left(\mathrm{Opd}_{\infty}^{\mathrm{ns}, \mathrm{gen}}\right)_{/ \cdot}^{\mathrm{op}}\right)^{\amalg} \rightarrow\left(\left(\mathrm{Opd}_{\infty}^{\mathrm{ns}, \mathrm{gen}}\right)^{\mathrm{op}}\right)^{\amalg}$. Combined with the lax monoidal algebra functor

$$
\left(\left(\mathrm{Opd}_{\infty}^{\mathrm{ns}, \mathrm{gen}}\right)^{\mathrm{op}}\right)^{\amalg} \times_{\mathbb{T} \text { op }}\left(\mathrm{Opd}_{\infty}^{\mathrm{ns,gen}}\right)^{\times} \rightarrow \mathrm{Cat}_{\infty}^{\times}
$$

of [Hau14b, Definition A.65], this gives a map of $\infty$-operads

$$
\left(\left(\operatorname{Opd}_{\infty}^{\text {ns,gen }}\right)_{/ \bullet}^{\text {op }}\right)^{\amalg} \times_{\mathbb{\llbracket} \text { op }}\left(\operatorname{Opd}_{\infty}^{\text {ns,gen }}\right)^{\times} \rightarrow\left(\left(\operatorname{Opd}_{\infty}^{\text {ns,gen }}\right)^{\text {op }}\right)^{\amalg} \times_{\mathbb{\llbracket} \text { op }}\left(\operatorname{Opd}_{\infty}^{\text {ns,gen }}\right)^{\times} \rightarrow \text { Cat }_{\infty}^{\times},
$$

with lax monoidal structure given, for $\mathcal{P}, \mathcal{Q}$ generalized non-symmetric $\infty$-operads over $\mathcal{O}$, by

$$
\operatorname{Alg}_{\mathcal{P}}(\mathcal{V}) \times \operatorname{Alg}_{\mathcal{Q}}(\mathcal{V}) \rightarrow \operatorname{Alg}_{\mathcal{P} \times \triangle^{\text {op }} \mathcal{Q}}(\mathcal{V}) \rightarrow \operatorname{Alg}_{\mathcal{P} \times \mathcal{O} Q}(\mathcal{V})
$$

Construction 9.9. The functors ${\triangle_{-,, \ldots,-}^{\mathrm{op}}}_{\mathcal{S}} \times(n+1) \rightarrow\left(\mathrm{Opd}_{\infty}^{\mathrm{ns}, \mathrm{gen}}\right)_{/ \triangle_{/[n]}^{\mathrm{op}}}$ preserve products, and considering their naturality in $[n] \in \triangle$ this gives rise to a natural transformation $\Delta^{\mathrm{op}} \times \Delta^{1} \rightarrow$ Cat ${ }_{\infty}^{\text {prod }}$ of functors from $\Delta^{\text {op }}$ to the $\infty$-category Cat $_{\infty}^{\text {prod }}$ of $\infty$-categories with products (and product-preserving functors). Here the functor

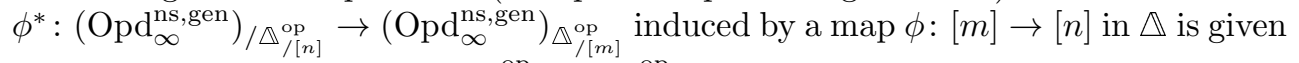
by taking pullbacks along $\phi_{*}: \mathbb{\triangle}_{/[m]}^{\mathrm{op}} \rightarrow \mathbb{\Delta}_{/[n]}^{\mathrm{op}}$.

By [Lur14, Corollary 2.4.1.9], the $\infty$-category Cat ${ }_{\infty}^{\text {prod }}$ is equivalent to the full subcategory of the $\infty$-category $\operatorname{Alg}_{\llbracket \text { op }}^{\Sigma}\left(\mathrm{Cat}_{\infty}\right)$ of symmetric monoidal $\infty$-categories 
(and symmetric monoidal functors) spanned by the Cartesian symmetric monoidal $\infty$-categories. Thus we get a map

$$
\Delta^{\mathrm{op}} \times \Delta^{1} \rightarrow \mathrm{Cat}_{\infty}^{\mathrm{prod}} \rightarrow \operatorname{Alg}_{\text {ॠop }}^{\Sigma}\left(\mathrm{Cat}_{\infty}\right)
$$

Since $(-)^{\mathrm{op}}: \mathrm{Cat}_{\infty} \rightarrow \mathrm{Cat}_{\infty}$ preserves products, it induces a functor

$$
(-)^{\mathrm{op}}: \operatorname{Alg}_{\llbracket \text { op }}^{\Sigma}\left(\mathrm{Cat}_{\infty}\right) \rightarrow \operatorname{Alg}_{\llbracket \text { op }}^{\Sigma}\left(\mathrm{Cat}_{\infty}\right)
$$

Combining this with the forgetful functor from symmetric monoidal $\infty$-categories to coCartesian fibrations over $\mathbb{T}^{\mathrm{op}}$, we get a functor

$$
\Delta^{\mathrm{op}} \times \Delta^{1} \rightarrow \operatorname{Alg}_{\llbracket^{\mathrm{op}}}^{\Sigma}\left(\mathrm{Cat}_{\infty}\right) \stackrel{(-)^{\mathrm{op}}}{\longrightarrow} \operatorname{Alg}_{\varpi^{\mathrm{opp}}}^{\Sigma}\left(\mathrm{Cat}_{\infty}\right) \rightarrow \mathrm{Cat}_{\infty / \mathbb{T}^{\mathrm{op}}}^{\mathrm{coCart}} \simeq \mathrm{Fun}\left(\llbracket^{\mathrm{op}}, \mathrm{Cat}_{\infty}\right) .
$$

Equivalently, this is a functor $\Delta^{1} \rightarrow \operatorname{Fun}\left(\Delta^{\mathrm{op}} \times \mathbb{\pi}^{\mathrm{op}}, \mathrm{Cat}_{\infty}\right)$, or a morphism

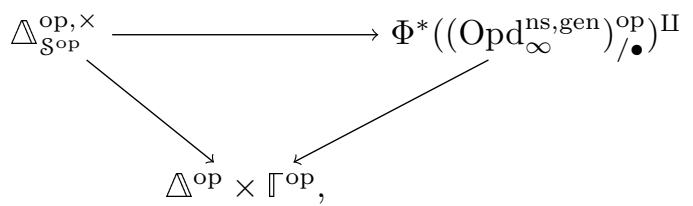

of coCartesian fibrations over $\triangle^{\mathrm{op}} \times \mathbb{\pi}^{\mathrm{op}}$, where

$$
\Phi^{*}\left(\left(\mathrm{Opd}_{\infty}^{\mathrm{ns}, \mathrm{gen}}\right)_{/ \bullet}^{\mathrm{op}}\right)^{\amalg} \rightarrow \Delta^{\mathrm{op}} \times \pi^{\mathrm{op}}
$$

denotes the pullback of $\left(\left(\mathrm{Opd}_{\infty}^{\mathrm{ns}, \mathrm{gen}}\right)_{/ \bullet}^{\mathrm{op}}\right)^{\amalg} \rightarrow\left(\mathrm{Opd}_{\infty}^{\mathrm{ns}, \mathrm{gen}}\right)^{\mathrm{op}} \times \mathbb{\llbracket}^{\mathrm{op}}$ along

$$
\Phi:=\triangle_{/(-)}^{\mathrm{op}} \times \mathrm{id}: \triangle^{\mathrm{op}} \times \mathbb{\square}^{\mathrm{op}} \rightarrow\left(\mathrm{Opd}_{\infty}^{\mathrm{ns}, \mathrm{gen}}\right)^{\mathrm{op}} \times \mathbb{\llbracket}^{\mathrm{op}} .
$$

Composing with the projection $\Delta^{\mathrm{op}} \times \mathbb{\square}^{\mathrm{op}} \rightarrow \mathbb{\llbracket}^{\mathrm{op}}$, we get in particular a morphism of generalized $\infty$-operads

$$
\triangle_{\text {gop }^{\mathrm{op}}}^{\mathrm{op}, \times} \rightarrow \Phi^{*}\left(\left(\mathrm{Opd}_{\infty}^{\mathrm{ns}, \mathrm{gen}}\right)_{/ \bullet}^{\mathrm{op}}\right)^{\amalg} \rightarrow\left(\left(\mathrm{Opd}_{\infty}^{\mathrm{ns}, \text { gen }}\right)_{/ \bullet}^{\mathrm{op}}\right)^{\amalg} .
$$

This sends an object $\left(\left(X_{0}^{1}, \ldots, X_{n}^{1}\right), \ldots,\left(X_{0}^{m}, \ldots, X_{n}^{m}\right)\right)$ in the fibre over $([n],\langle m\rangle)$ to $\left(\triangle_{X_{0}^{1}, \ldots, X_{n}^{1}}^{\mathrm{op}}, \ldots, \triangle_{X_{0}^{m}, \ldots, X_{n}^{m}}^{\mathrm{op}}\right)$.

Construction 9.10. Combining Constructions 9.8 and 9.9, we get a morphism of generalized $\infty$-operads

$$
{\triangle_{\mathcal{S}}^{\mathrm{op}}}_{\mathrm{op}, \times} \times_{\llbracket \text { op }}\left(\mathrm{Opd}_{\infty}^{\mathrm{ns}, \mathrm{gen}}\right)^{\times} \rightarrow\left(\left(\mathrm{Opd}_{\infty}^{\mathrm{ns}, \mathrm{gen}}\right)_{/ \bullet}^{\mathrm{op}}\right)^{\times} \times_{\llbracket \text { op }}\left(\mathrm{Opd}_{\infty}^{\mathrm{ns}, \mathrm{gen}}\right)^{\times} \rightarrow \mathrm{Cat}_{\infty}^{\times}
$$

By (a slight variant of) [Lur14, Proposition 2.4.2.5] this corresponds to a monoid object $\triangle_{\text {Sop }}^{\text {op }} \times \times_{\mathbb{T} \text { op }}\left(\operatorname{Opd}_{\infty}^{\text {ns,gen }}\right)^{\times} \rightarrow \mathrm{Cat}_{\infty}$. Let

$$
\left(\overline{\mathfrak{A L G}}_{\text {cat }}^{\otimes}\right)^{\vee} \rightarrow\left(\triangle_{\text {gop }}^{\text {op, } \times} \times_{\mathbb{T}_{\text {op }}}\left(\operatorname{Opd}_{\infty}^{\text {ns,gen }}\right)^{\times}\right)^{\text {op }}
$$

be a Cartesian fibration associated to this functor. Then the composite

$$
\begin{aligned}
& \left(\overline{\mathfrak{A L G}}_{\text {cat }}^{\otimes}\right)^{\vee} \rightarrow\left(\triangle_{\mathcal{S}^{\text {op }}}^{\text {op } \times} \times_{\text {oop }}\left(\mathrm{Opd}_{\infty}^{\text {ns,gen }}\right)^{\times}\right)^{\text {op }} \\
& \rightarrow\left(\left(\Delta^{\mathrm{op}} \times \mathbb{\mathbb { T }}^{\mathrm{op}}\right) \times_{\mathbb{J} \mathrm{op}}\left(\mathrm{Opd}_{\infty}^{\mathrm{ns}, \mathrm{gen}}\right)^{\times}\right)^{\mathrm{op}} \\
& \simeq\left(\triangle^{\mathrm{op}} \times\left(\mathrm{Opd}_{\infty}^{\mathrm{ns}, \mathrm{gen}}\right)^{\times}\right)^{\mathrm{op}}
\end{aligned}
$$

is again a Cartesian fibration, and we define $\overline{\mathfrak{A L G}}_{\text {cat }}^{\otimes} \rightarrow \Delta^{\mathrm{op}} \times\left(\mathrm{Opd}_{\infty}^{\text {ns,gen }}\right)^{\times}$to be 
a coCartesian fibration associated to this Cartesian fibration. This is a coCartesian fibration of generalized non-symmetric $\infty$-operads.

Construction 9.11. The symmetric monoidal structure on $\widehat{\mathrm{Cat}}_{\infty}^{\mathrm{coC}}$ induces a tensor product on $\mathrm{Mon}_{\infty}^{\mathrm{coC}}$, and the forgetful functor from $\mathrm{Mon}_{\infty}^{\mathrm{coC}}$ to $\widehat{\mathrm{Opd}}_{\infty}^{\mathrm{ns}, \mathrm{gen}}$ can be enhanced to a lax monoidal functor $\operatorname{Mon}_{\infty}^{\mathrm{coC}, \otimes} \rightarrow\left(\widehat{\mathrm{Opd}}_{\infty}^{\mathrm{ns}, \mathrm{gen}}\right)^{\times}$. Let $\left({\overline{\mathfrak{A} \mathfrak{L} \mathfrak{G}_{\text {cat }}}}^{\otimes}\right)^{\prime} \rightarrow$ $\triangle^{\mathrm{op}} \times \operatorname{Mon}_{\infty}^{\mathrm{coC}, \otimes}$ be defined by the pullback

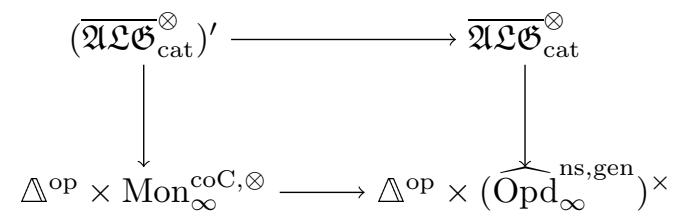

along this, where the right vertical map is the variant of the map of Construction 9.10 where we allow the target generalized non-symmetric $\infty$-operads to be large. This gives a coCartesian fibration of generalized non-symmetric $\infty$-operads.

Definition 9.12. We define $\mathfrak{A} \mathfrak{L G}_{\text {cat }}^{\otimes}$ to be the full subcategory of $\left({\overline{\mathfrak{A} \mathfrak{L} \mathfrak{G}_{\text {cat }}}}^{\otimes}\right)^{\prime}$ spanned by those objects that correspond to lists of objects of $\mathfrak{A} \mathfrak{L} \mathfrak{G}_{\text {cat }}$.

Proposition 9.13. The restricted projection $\mathfrak{A L G}_{\text {cat }}^{\otimes} \rightarrow \mathbb{\Delta}^{\mathrm{op}} \times \mathrm{Mon}_{\infty}^{\mathrm{coC}, \otimes}$ is a coCartesian fibration.

To see this we use the following technical observation:

Lemma 9.14. Let $X_{0}, \ldots, X_{n}$ and $Y_{0}, \ldots, Y_{n}$ be spaces, and suppose $(\Xi, \mathrm{H})$ is an object of $\triangle_{X_{0} \times Y_{0}, \ldots, X_{n} \times Y_{n}}^{\text {op }}$ over $\xi \in \mathbb{\Delta}_{/[n]}^{\mathrm{op}}$. Then the map

$$
\left(\mathbb{\Lambda}_{X_{0} \times Y_{0}, \ldots, X_{n} \times Y_{n}}^{\text {op }}\right)_{/(\Xi, \mathrm{H})}^{\text {act }} \rightarrow\left(\mathbb{\Lambda}_{X_{0}, \ldots, X_{n}}^{\text {op }}\right)_{/ \Xi}^{\text {act }} \times\left(\mathbb{N}_{X_{0}, \ldots, X_{n}}^{\text {op }}\right)_{/ \mathrm{H}}^{\text {act }}
$$

is cofinal.

Proof. We have a pullback square

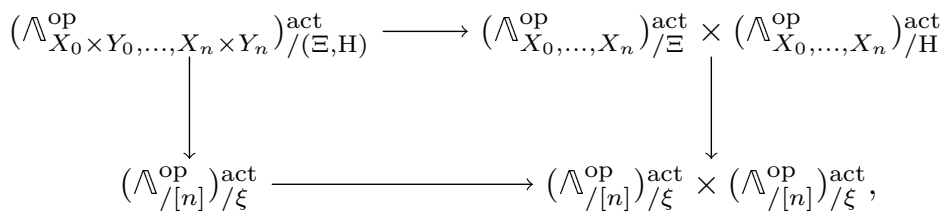

where the vertical maps are left fibrations by Lemma 6.7, and the bottom horizontal map is cofinal since the $\infty$-category $\left({\left.\bigwedge_{/[n]}^{\mathrm{op}}\right)}_{/ \xi}^{\text {act }}\right.$ is sifted by [Hau14b, Lemma 4.17]. It therefore follows from [Lur09, Proposition 4.1.2.15] and [Lur09, Remark 4.1.2.10] that the top horizontal map is also cofinal.

Proof of Proposition 9.13. It suffices to show that given a pair of composite algebras $M: \mathbb{\triangle}_{X_{0}, \ldots, X_{n}}^{\mathrm{op}} \rightarrow \mathcal{V}^{\otimes}$ and $N: \triangle_{Y_{0}, \ldots, Y_{n}}^{\mathrm{op}} \rightarrow \mathcal{W}^{\otimes}$, their external product

$$
M \otimes N: \triangle_{X_{0} \times Y_{0}, \ldots, X_{n} \times Y_{n}}^{\mathrm{op}} \rightarrow \mathcal{V}^{\otimes} \times_{\Delta^{\mathrm{op}}} \mathcal{W}^{\otimes}
$$

is also composite. This follows from Lemma 9.14 together with the definition of left operadic Kan extensions. 
Corollary 9.15. $\mathfrak{A} \mathfrak{L} \mathfrak{G}_{\text {cat }}^{\otimes}$ determines a lax monoidal functor

$$
\operatorname{Mon}_{\infty}^{\mathrm{coC}, \otimes} \rightarrow \operatorname{Cat}\left(\mathrm{Cat}_{\infty}\right)^{\times} .
$$

Corollary 9.16. Suppose $\mathcal{O}$ is a (symmetric) $\infty$-operad and $\mathcal{V}$ is an $\mathcal{O} \otimes \mathbb{E}_{1}$-monoidal $\infty$-category compatible with small colimits. Then the double $\infty$-category $\mathfrak{A} \mathfrak{L G}_{\text {cat }}(\mathcal{V})$ inherits a natural $\mathcal{O}$-monoidal structure.

Proof. By the universal property of the Boardman-Vogt tensor product of $\infty$-operads, we can identify an $\mathcal{O} \otimes \mathbb{E}_{1}$-monoidal $\infty$-category compatible with small colimits with an $\mathcal{O}$-algebra in $\mathrm{Mon}_{\infty}^{\mathrm{coC}}$. By Corollary 9.15, this implies that the functor $\mathfrak{A} \mathfrak{L} \mathfrak{G}_{\text {cat }}$ takes $\mathcal{V}$ to an $\mathcal{O}$-algebra in double $\infty$-categories, i.e. an $\mathcal{O}$-monoidal double $\infty$-category.

Lurie's additivity theorem, [Lur14, Theorem 5.1.2.2], gives an equivalence

$$
\mathbb{E}_{n} \otimes \mathbb{E}_{1} \stackrel{\sim}{\rightarrow} \mathbb{E}_{n+1},
$$

so as a special case we have:

Corollary 9.17. Suppose $\mathcal{V}$ is an $\mathbb{E}_{n+1}$-monoidal $\infty$-category compatible with small colimits. Then the double $\infty$-category $\mathfrak{A} \mathfrak{L G}_{\text {cat }}(\mathcal{V})$ inherits a natural $\mathbb{E}_{n}$-monoidal structure.

Taking the colimit of these equivalences as $n \rightarrow \infty$, we get an equivalence

$$
\mathbb{E}_{\infty} \otimes \mathbb{E}_{1} \stackrel{\sim}{\rightarrow} \mathbb{E}_{\infty}
$$

which similarly gives:

Corollary 9.18. Suppose $\mathcal{V}$ is a symmetric monoidal $\infty$-category compatible with

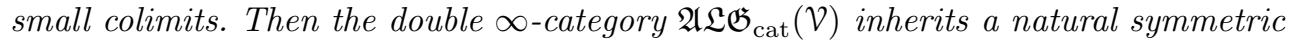
monoidal structure.

\section{References}

[Bac10] Hugo V. Bacard, Segal enriched categories I (2010), available at arXiv:1009.3673.

[GH15] David Gepner and Rune Haugseng, Enriched $\infty$-categories via non-symmetric $\infty$-operads, Adv. Math. 279 (2015), 575-716, available at arXiv:1312.3178.

[GHN15] David Gepner, Rune Haugseng, and Thomas Nikolaus, Lax colimits and free fibrations in $\infty$-categories (2015), available at arXiv:1501.02161.

[Hau14a] Rune Haugseng, Iterated spans and "classical" topological field theories (2014), available at arXiv: 1409.0837.

[Hau14b] - The higher Morita category of $E_{n}$-algebras (2014), available at arXiv: 1412.8459.

[Lur09] Jacob Lurie, Higher Topos Theory, Annals of Mathematics Studies, vol. 170, Princeton University Press, Princeton, NJ, 2009. Available at http://math.harvard.edu/ lurie/ papers/highertopoi.pdf.

[Lur14] _ Higher Algebra, 2014. Available at http://math.harvard.edu/ lurie/papers/ higheralgebra.pdf.

Rune Haugseng haugseng@mpim-bonn.mpg.de

Max-Planck-Institut für Mathematik, Vivatsgasse 7, 53111 Bonn, Germany 